\title{
The relationship between first-level leadership and inner-context and implementation outcomes in behavioral health: a scoping review
}

Rosemary D. Meza ${ }^{*}$ D, Noah S. Triplett, Grace S. Woodard, Prerna Martin, Alya N. Khairuzzaman, Gabrielle Jamora and Shannon Dorsey

\begin{abstract}
Background: First-level leadership is uniquely positioned to support evidence-based practice (EBP) implementation for behavioral health due to first-level leaders' access to and relationship with service providers. First-level leaders are individuals who directly supervise and manage frontline employees who do not manage others. However, firstlevel leadership is underrepresented in existing reviews of the impact of leadership on EBP implementation. This review describes the relationship between first-level leadership and implementation determinants and outcomes.

Methods: A scoping review was performed to synthesize the literature on the relationship between first-level leadership and inner-context and implementation outcomes. A literature search was conducted in PubMed, Eric, PsycINFO, CINAHL, Scopus, and Web of Science. To be eligible, studies had to examine first-level leadership, be conducted in settings providing behavioral health services, and examine the relationship between first-level leadership and an implementation or inner-context outcome. Data extraction and synthesis were performed to describe study characteristics, leader-outcome relationships, and overlap in leadership frameworks.

Results: Twenty-one records met our inclusion criteria. Studies primarily relied on observational designs and were often cross-sectional. Studies more often examined general leadership rather than leadership strategically focused on EBP implementation (i.e., strategic implementation leadership). Our findings suggest that several forms of firstlevel leadership are inconsistently related to a broad set of implementation determinants, with infrequent examination of specific implementation outcomes. The broad set of implementation determinants studied, limited number of replications, and inconsistent findings have resulted in sparse evidence for any specific leadershipoutcome relationship. The greatest accumulation of evidence exists for general leadership's positive relationship with providers' EBP attitudes, most notably in the form of transformational leadership. This was followed by evidence for strategic implementation leadership facilitating general implementation. Our synthesis revealed moderate conceptual overlap of strategic implementation leadership behaviors described in the theory of implementation leadership and theory of middle managers' role in implementation.

(Continued on next page)
\end{abstract}

\footnotetext{
* Correspondence: rdmeza@uw.edu

Department of Psychology, University of Washington, Guthrie Hall 119A, Box 351525, Seattle, WA 98195, USA
}

C C The Author(s). 2021 Open Access This article is licensed under a Creative Commons Attribution 4.0 International License, which permits use, sharing, adaptation, distribution and reproduction in any medium or format, as long as you give appropriate credit to the original author(s) and the source, provide a link to the Creative Commons licence, and indicate if changes were made. The images or other third party material in this article are included in the article's Creative Commons licence, unless indicated otherwise in a credit line to the material. If material is not included in the article's Creative Commons licence and your intended use is not permitted by statutory regulation or exceeds the permitted use, you will need to obtain permission directly from the copyright holder. To view a copy of this licence, visit http://creativecommons.org/licenses/by/4.0/ The Creative Commons Public Domain Dedication waiver (http://creativecommons.org/publicdomain/zero/1.0/) applies to the data made available in this article, unless otherwise stated in a credit line to the data. 
(Continued from previous page)

Conclusions: Our findings suggest that first-level leadership may play an important role in shaping implementation determinants and outcomes, but consistent empirical support is sparse and confidence dampened by methodological issues. To advance the field, we need studies that adopt stronger methodological rigor, address the conceptual overlap in leadership frameworks, examine a broader set of implementation outcomes, and examine conditions under which leadership impacts implementation.

Trial registration: This review was not registered.

Keywords: First-level leadership, Implementation outcomes, Implementation determinants, Inner-context outcomes, Behavioral health

\section{Contributions to the literature}

- This is the first scoping review to explore how first-level leadership shapes implementation determinants and outcomes for behavioral health services.

- The positive relationship between first-level transformational leadership and providers' EBP attitudes was a primary focus of studies; however, the evidence linking provider attitudes to implementation outcomes is, at best, mixed.

- There was very limited evidence linking any form of firstlevel leadership to a specific implementation outcome and findings were inconsistent.

- Our qualitative synthesis suggests that there is significant conceptual overlap in behaviors described in the theories of implementation leadership and middle managers' role in implementation, highlighting the need to disentangle how these strategic forms of implementation leadership uniquely relate to EBP implementation.

- We offer conceptual and methodological guidance for the field to advance our understanding of whether and how first-level leadership supports EBP implementation.

\section{Introduction}

Despite advances in evidence-based practices (EBPs) to treat mental and behavioral health problems [1], efforts to transfer them from laboratory to public settings are often unsuccessful [2, 3]. Implementation frameworks suggest that leadership may function as a mechanism for improving EBP implementation [4-7]. Empirical studies support this theoretical link between leadership and the innovation implementation in healthcare settings [8-10]. While there is no universally adopted leadership definition, conceptualizations generally reflect a process of intentional efforts by an individual to motivate, influence, and enable a person or group of people with the aim of impacting group or organizational outcomes [11-13]. Leadership across organizational levels-from top leaders who establish organizational policies and practices [14] to first-level leaders who directly supervise employees providing direct services-and their alignment with one another influence the success of implementation $[15,16]$. Achieving organizational effectiveness, including effectiveness in EBP implementation, is complex and hinges on coordination across levels of leadership [16, 17]. Organizational leadership at the first-level may be particularly influential in supporting EBP implementation due to leaders' access to and direct relationships with service providers. For instance, firstlevel leadership may function to support or hinder the realization of top-level leaders' organizational policies that are favorable for EBP implementation. Conversely, first-level leadership may mitigate potential negative impacts of organizational policies instituted by top leaders that would hinder implementation.

First-level leaders are individuals who directly supervise and manage frontline employees who do not manage others [18]. In the context of behavioral health service delivery, first-level leaders tend to include clinical supervisors, program managers, and team leaders who supervise direct providers. Leaders at this lowest level enact their leadership influence through direct interaction with frontline employees, making decisions that concern day-to-day work, anticipating and solving current problems, and using practical judgment to address ongoing problems [19]. They engage and inspire staff at the frontline [17]. In the context of implementation, first-level leadership is theorized to improve general and strategic cultures and climates, provider attitudes, and implementation outcomes $[4,16,20]$.

There have been calls for leadership to be studied within a particular organizational level and context because the antecedents, consequences, and dynamics of leadership change as a function of organizational level, structure, and complexity [19]. The role of first-level leadership differs from leadership at higher organizational levels. At the highest level, leaders are responsible for establishing a comprehensive direction for the organization, creating organizational policy, and crafting organizational strategy [19]. Mid-level leaders engage in coordination of multiple subunits, manage less directly, and are responsible for establishing operational 
practices and policies [19]. The success of innovation implementation relies on numerous complex factors, some of which include training, teaching and feedback, time requirements, reimbursement, resource allocation, and workflow integration $[4,5]$. Leaders across organizational levels are differentially positioned to impact these factors, which has implications for their influence on implementation. Given the distinct responsibilities of firstlevel leaders focused on day-to-day decisions and problem solving, synthesis of the literature is needed to understand how first-level leadership influences implementation and what implementation determinants and outcomes they impact.

Empirical research examining leadership and training programs to develop effective leadership have lagged in behavioral health relative to health broadly. The number of studies examining the role of leadership in behavioral health innovation implementation has not matched that of health innovations broadly [8]. Moreover, first-level leaders within behavioral health tend to be promoted internally due to their effectiveness as providers, thus are often unprepared and lacking formal leadership training $[21,22]$. While leadership training is a continued need in healthcare broadly, many examples of formal training programs have existed in medicine for upwards of 20 years [23-26]. Efforts to train leaders in behavioral health, including those with a focus on EBP implementation, are limited (e.g., $[27,28])$. Synthesis of the literature is needed to understand the type of leadership styles and behaviors those in behavioral health draw on to support implementation. Further, implementation determinants and outcomes are theorized to interact with innovation characteristics [4, 29]. Behavioral health has followed the lead of evidence-based medicine in using research evidence to standardize healthcare [30] yielding an abundance of beneficial EBPs. Attending to and measuring delivery of EBPs is one important method by which leaders can impact EBT delivery [16]. However, monitoring and evaluating the content and dose of complex behavioral health interventions is difficult [31]. Efforts to standardize delivery and maintain quality have included detailed treatment manuals, training, certification requirements, and fidelity criteria [32], yet modification of behavioral health interventions is common [33] and direct observation to monitor and support delivery is often unfeasible [34]. Further, many treatments are based on principles of practice rather than prescribed strategies (e.g., [35-37]) posing additional challenges to defining and monitoring their adoption, fidelity, and sustained use. These characteristics of behavioral health interventions may shape which first-level leadership behaviors are most effective in impacting implementation and the degree to which first-level leadership is able to impact implementation. For instance, task-oriented leadership behaviors such as planning and monitoring performance [38] may be more effective in ensuring behavioral health interventions are adopted and used with high fidelity than change-oriented behaviors primarily concerned with providing vision and encouraging innovation [38].

First-level leaders may influence implementation through leadership that aims to impact performance outcomes generally (i.e., general leadership) and through leadership that is strategically focused on influencing implementation outcomes (i.e., strategic implementation leadership). The full range leadership model, one of the most studied models of general leadership, describes three primary forms of leadership, transformational, transactional, and laissez-faire leadership [39]. Transformational leadership describes how leaders inspire and motivate employees to perform beyond expectations. Transactional leadership functions through reinforcement and exchanges, where leaders reward employees who fulfill expectations [39-41]. Passiveavoidant leadership is a style of non-leadership [42], characterized as taking a "hands off" approach by altogether avoiding making decisions or managing employees [43]. A recent systematic review found that managers engage in behaviors that are consistent with transformational and transactional approaches to support research use by clinical staff in nursing and allied health professionals [44].

Leadership that is strategically focused on EBP implementation is theorized to promote a positive climate for implementation and, in turn, foster positive attitudes toward EBPs that support implementation [45]. The implementation leadership theory [46] and theory of middle managers' role in implementation $[47,48]$ describe leadership behaviors that strategically focus on implementation among first-level leaders. Implementation leadership describes first-level leadership that proactively anticipates and addresses implementation challenges, demonstrates a deep understanding of EBP implementation, perseveres through implementation challenges, and supports providers to adopt and use EBPs [46]. The theory of middle managers' role in implementation is based on review and research on both first-level leaders and mid-level managers [4749]. It describes middle manager commitment to innovation implementation operationalized as four ways that managers can demonstrate their commitment for innovation implementation: obtaining and diffusing information about an innovation, adapting information and the innovation, mediating between strategy and day-to-day activities, and selling innovation implementation. In healthcare settings, there is some evidence suggesting that middle manager commitment to implementation-in conjunction with executive support for 
implementation and access to human resources administration-is associated with implementation effectiveness [50].

\section{Rationale for the scoping review}

Our overarching goal is to synthesize the literature examining how first-level leadership impacts implementation; therefore, we focus on implementation outcomes and determinants. First-level leadership, being situated within the organization's inner-context, is most suited to impact determinants within the inner-context as described in the Exploration, Preparation, Implementation, and Sustainment (EPIS) framework [4, 29]. These determinants include organizational characteristics, quality and fidelity monitoring and support, organizational staffing processes, and individual adopter characteristics. Similar to the general and strategic focus of leadership, implementation determinants in the inner-context can also be general and strategic. For instance, organizational cultures and climates reflect general organizational characteristics while implementation climate is an organizational characteristic strategically focused on implementation. We focus on inner-context outcomes in addition to implementation outcomes because many inner-context factors function as determinants of implementation outcomes [51, 52].

\section{Current study}

This scoping review aimed to summarize the existing research examining how first-level leadership relates to inner-context and implementation outcomes for behavioral health services. We aimed to clarify key concepts around how leadership has been defined and studied, identify gaps in the knowledge base, and report on the types of evidence that have informed this field [53]. We also aimed to identify future directions to guide this body of research.

\section{Method}

We followed Arksey and Malley's [54] methodological framework for scoping reviews. A scoping review was conducted due to the broad, emerging state of literature that cannot be subject to the narrow systematic review criteria due to its heterogenous nature [55]. We followed the preferred reporting items for systematic reviews and meta-analyses extension for scoping reviews (PRISMA$\mathrm{ScR})$ to enhance transparency. There is no registered review protocol. Additional file 1 includes the PRISMAScR checklist and Fig. 1 contains the PRISMA diagram.

\section{Search strategy}

We developed a comprehensive search strategy with a health sciences librarian. To ensure the most relevant articles were identified, we reviewed the title, abstract, and keywords from preselected articles to generate a list of appropriate search terms. Subject headings and keywords were identified and categorized into the following groups (1) implementation; (2) leader, supervisor, manager, or other synonym; (3) intervention; and (4) mental and behavioral health services (see Additional file 2 for search terms). The search was conducted in June 2019 using the following databases: PubMed, Eric, PsycINFO, CINAHL, Scopus, and Web of Science. We also examined the reference lists of studies to identify additional articles. Two additional studies were identified through the Implementation Science journal between the initial search and February 2020.

\section{Study inclusion and exclusion criteria}

Studies were included if they (1) examined the leadership of first-level leaders; (2) were conducted in settings providing behavioral health services; and (3) examined the influence of first-level leadership on an implementation outcome or inner-context outcome based on definitions of inner-context outcomes in the EPIS framework [29] (see Table 6) and implementation outcomes defined by Proctor and colleagues [63]. Studies that measured perceptions of "implementation" as an outcome, which we term "general implementation," were included if their measurement of implementation conceptually aligned with one or more implementation outcomes [63] (e.g., adoption and fidelity). Studies were excluded if leadership could not be clearly attributed to a first-level leader such as using the term "leader," "manager," or "management" without identifying their responsibility for direct supervision of front-line staff. Studies were considered to be providing behavioral health services if they described primarily delivering mental and behavioral health treatments (e.g., addiction health services, mental health services). Studies could be situated in settings that provide a variety of services (e.g., hospitals, human service agencies) if they were examining the delivery of behavioral health services. Review articles without original data, non-empirical studies, and study protocols were excluded. Articles were limited to those published in peer-reviewed journals in English. No restrictions were imposed on publication date, study design, or length of follow-up.

\section{Study selection and quality assessment}

Five reviewers (RM, NST, GSW, AK, GJ) assessed studies independently to determine inclusion status. Titles and abstracts were independently assessed by two reviewers, and each pair of reviewers met to discuss discrepancies. When necessary, consensus was reached with a third reviewer. This process was repeated for fulltext review. Consistent with the guidance on conducting scoping reviews, we did not assess the methodological 


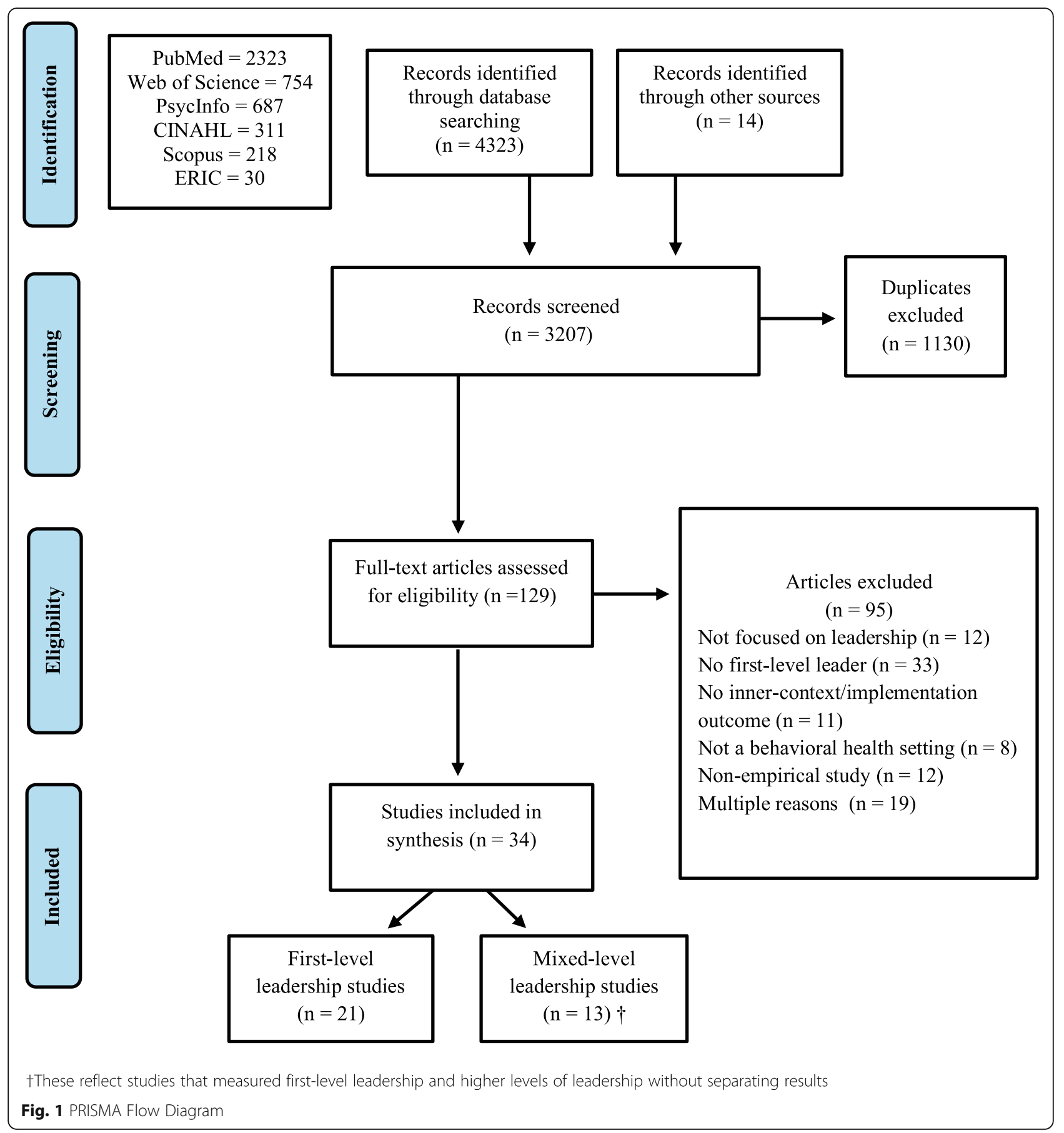

quality or risk of bias of the included articles [53]. Our goal was to provide an overview of the existing evidence base regardless of study quality.

\section{Data extraction}

A draft charting table was developed prior to extraction to capture study characteristics and key findings. The charting table was refined as reviewers trialed the extraction on a small sample of articles. Pairs of reviewers extracted data from each article and discussed discrepancies until they reached consensus. When necessary, a third reviewer was consulted to reach consensus. The extracted data items are listed in Table 1. The extracted items captured first-level leaders' behaviors, characteristics and/or leadership style examined, inner-context and/or 
Table 1 Data extraction

\begin{tabular}{|c|c|}
\hline Information extracted & Description \\
\hline Author & List of authors \\
\hline Year & Publication year \\
\hline Title & Study title \\
\hline Country & Country where the study was conducted \\
\hline Setting & $\begin{array}{l}\text { Physical location where the study was conducted (e.g., } \\
\text { mental health agency) }\end{array}$ \\
\hline Design & $\begin{array}{l}\text { Study design as reported or inferred from study methods } \\
\text { (e.g., observational, experimental) }\end{array}$ \\
\hline Study methodology & $\begin{array}{l}\text { Study methodology (qualitative, quantitative, mixed } \\
\text { methods) }\end{array}$ \\
\hline Data sources & Types of instruments used (e.g., survey, interview) \\
\hline Measures & Names of measures used and respondents \\
\hline Construct validity & $\begin{array}{l}\text { Whether or not measures had established construct } \\
\text { validity }\end{array}$ \\
\hline EBP or clinical innovation & EBP or clinical innovation being implemented \\
\hline Phase of implementation & $\begin{array}{l}\text { Stage of the EBP implementation (exploration, preparation, } \\
\text { implementation, sustainment, multiple, no active } \\
\text { implementation, or not reported) }\end{array}$ \\
\hline Leader role & $\begin{array}{l}\text { Level of leadership included and leaders' role in supporting } \\
\text { EBP delivery or implementation }\end{array}$ \\
\hline First-level leadership & Leadership style, behaviors or characteristics examined \\
\hline Inner-context outcomes & EPIS inner-context factors examined \\
\hline Implementation outcomes & Implementation outcomes examined \\
\hline Level of analysis & $\begin{array}{l}\text { Level at which studies measured and analyzed leadership and } \\
\text { inner-context and implementation outcomes (individual, team, } \\
\text { organization) }\end{array}$ \\
\hline Results & $\begin{array}{l}\text { Description of the nature of the associations between } \\
\text { leadership and inner-context and implementation outcomes }\end{array}$ \\
\hline
\end{tabular}

EBP evidence-based practice, EPIS exploration, preparation, implementation, sustainment framework

implementation outcomes studied, study characteristics, and key findings.

\section{Data synthesis}

Synthesis involved quantitative analysis (e.g., descriptive statistics) of study characteristics and qualitative analysis of the leader behaviors and characteristics measured in included studies. We categorized inner-context outcomes based on the original and expanded EPIS framework [4, 29]. We also categorized findings related to implementation outcomes based on Proctor and colleagues [63] framework.

We calculated frequencies to describe the number of studies examining the relationship between leadership and inner-context outcomes. We provide a descriptive summary of the relationship between leadership styles and inner-context and implementation outcomes reported. To do this, we coded results to reflect the direction of the relationship (i.e., positive, negative, or nonsignificant) reported in studies. A single study could yield multiple discrepant results (i.e., positive, negative, and/or non-significant) if they only measured subscales of a construct (e.g., subscales of transformational leadership). However, when studies reported total scores, we report those associations. The coded relationships include direct associations and indirect mediated associations between leadership and inner-context or implementation outcomes.

We used a deductive approach to code leader behaviors and characteristics in studies that qualitatively measured leadership. We operationalized leader behaviors as actions a leader engages in that may influence the implementation process (e.g., provision of implementation prescriptions, empowering staff) and operationalized leader characteristics as qualities, perceptions, or traits of a leader that may influence the process of implementation (e.g., enthusiasm, attitudes toward EBPs), adapted from Moullin and colleagues [29]. The codebook was developed by first incorporating the leadership styles included in this review's quantitative studies (e.g., transformational, implementation leadership). Then, we referenced reviews of leadership styles and leader 
behaviors from the organizational behavior, leadership, and middle manager literature to incorporate other styles of leadership not captured in this review's quantitative studies (e.g., EBP champion, authentic leadership) [49, 64-67]. When a leader behavior or characteristic did not fit with an existing leadership construct, it was included as a standalone code. Leader behaviors could be coded as reflecting more than one leadership style or framework (e.g., implementation leadership and middle managers' implementation roles). The percent of overlapping codes were calculated to identify construct overlap in the existing frameworks. All data synthesis was completed by two authors and a third author was consulted as needed.

\section{Results}

The search yielded a total of 4337 articles. After excluding duplicates, 3207 titles and abstracts were reviewed for inclusion. Among those, 129 articles progressed to full-text review and 21 met criteria for data abstraction (Fig. 1). Thirteen additional articles met criteria for abstraction [68-80], but combined their measurement of first-level and higher levels of leadership limiting conclusions about how first-level leadership specifically is related to implementation. Data were abstracted from these articles, but their results are not combined with the 21 primary articles and are listed in the supplemental materials for comparison (additional file 3 Tables 9-12).

\section{Study characteristics}

\section{Study methods}

Tables 2 and 3 describe the included studies and study characteristics. Studies were most commonly conducted in mental health agencies (81\%) and set in the USA (79\%). Most used an observational design (90\%) and were cross-sectional (57\%). Methods included quantitative (57\%), qualitative (29\%), and mixed methods (14\%). Most studies examined the relationship between leadership and an inner-context or implementation outcome while implementing a clinical innovation (62\%). Studies were mainly conducted during the implementation (52\%) phase.

Thirteen studies (62\%) used a questionnaire to measure first-level leadership and of those, 92\% used a leadership questionnaire with established construct validity. Fifteen studies (71\%) used a questionnaire to measure either or both inner-context and implementation outcomes. Of those fifteen studies, $73 \%$ relied fully on an instrument with established construct validity. Of the five studies measuring a specific implementation outcome, $60 \%$ relied on self-report from organizational members. Of the 13 quantitative studies, most (69\%) relied on a single respondent for independent and dependent measures.

Table 4 summarizes the level of analysis for leadership and outcomes. Studies tended to analyze perceptions of first-level leadership at the team (43\%) and organizationlevel (43\%). Inner-context outcomes were mainly studied at the individual-level (43\%) while implementation outcomes were primarily studied at the organization-level (43\%).

\section{Leaders and leadership styles examined}

Few studies described the assigned or intended role of the first-level leader in implementation (29\%). Descriptions of first-level leaders' roles were often vague, with the richest description stating, "leaders were required to manage the new programs, provide supervision, and also arrange financing for the program" [95].

A glossary of leadership constructs examined is included in Table 5. Most studies examined a general style of leadership (76\%). Transformational leadership (67\%) was the most common style of general leadership examined, followed by transactional (29\%) and passiveavoidant (19\%) leadership. Fifty-seven percent of studies examined leadership that strategically focused on implementation. These included implementation leadership (52\%), behaviors described in the theory of middle managers' role in implementation (29\%) [48], and leaders acting as EBP champions (10\%). While general leadership was most often measured by quantitative surveys, strategic implementation leadership was primarily measured through qualitative interviews. Additionally, studies described leader behaviors (26\%) and characteristics (5\%) that could not be otherwise categorized into existing leadership theories.

\section{Inner-context factors}

A glossary of inner-context factors examined is included in Table 6. Twelve studies (57\%) examined the relationship between leadership and two of the five broad inner-context constructs: individual characteristics (38\%) and organizational characteristics (38\%). Individual characteristics that were specifically focused on EBPs (i.e., providers' EBP attitudes and EBP knowledge) were more common than individual characteristics that were generally focused (i.e., providers' burnout). Providers' EBP attitudes was the most common inner-context outcome examined (24\%). Organizational characteristics had a slight tendency to be generally focused (i.e., organizational climate and organizational culture) rather than strategically focused (i.e., implementation climate and innovation climate). 
Table 2 Summary of included studies ( $N=21$ )

\begin{tabular}{|c|c|c|c|c|c|c|c|}
\hline $\begin{array}{l}\text { First } \\
\text { author }\end{array}$ & $\begin{array}{l}\text { Leadership } \\
\text { type(s) }\end{array}$ & $\begin{array}{l}\text { Inner-context } \\
\text { outcomes }\end{array}$ & IS outcomes & Design & Setting & IS phase & Key leadership findings \\
\hline $\begin{array}{l}\text { Aarons } \\
2006 \text { [81] }\end{array}$ & TfL, TrL & IC & N/a & Obs & $\mathrm{CMH}$ & $\mathrm{NI}$ & TrL and TfL positively predicted providers' EBPA. \\
\hline $\begin{array}{l}\text { Aarons } \\
\text { et al. } \\
2012[82]\end{array}$ & TfL & OC, IC; L & N/a & Exp & CW & । & $\begin{array}{l}\text { During EBP implementation, TfL positively predicted InnCli, } \\
\text { and InnCli positively predicted providers' EBPA. When } \\
\text { delivering services as usual, LMX mediated the relation } \\
\text { between leaders' TfL and InnCli. }\end{array}$ \\
\hline $\begin{array}{l}\text { Aarons } \\
\text { et al. } \\
2015[28]\end{array}$ & TfL & OC & N/a & Obs & $\mathrm{MH}$ & $\mathrm{NI}$ & $\begin{array}{l}\text { OrgCul suffered more where leaders rated their TfL more } \\
\text { positively than providers rated them, in contrast to where } \\
\text { leaders rated themselves lower than providers. OrgCul tended } \\
\text { to be better when providers and leaders agreed the leaders' } \\
\text { TfL was high than when they agreed it was low. }\end{array}$ \\
\hline $\begin{array}{l}\text { Aarons } \\
\text { et al. } \\
2016[83]\end{array}$ & $\begin{array}{l}\text { TfL, TrL; } \\
\text { PaL, LB }\end{array}$ & N/a & Sus & Obs & $\begin{array}{l}\mathrm{CMH} \\
\mathrm{CW} \\
\text { Oth }\end{array}$ & Sus & $\begin{array}{l}\text { TfL positively predicted sustainment, and PaL negatively } \\
\text { predicted sustainment. Leaders' ongoing championing of EBP } \\
\text { and practical support for providers facilitated sustainment. }\end{array}$ \\
\hline $\begin{array}{l}\text { Aarons } \\
\text { et al. } \\
2017[45]\end{array}$ & IL & OC & N/a & Obs & $\mathrm{MH}$ & $\mathrm{NI}$ & $\begin{array}{l}\text { OrgCli of involvement and performance feedback were } \\
\text { highest when leaders rated their IL low and providers rated } \\
\text { leaders' IL high. Involvement climate did not differ when } \\
\text { leaders and providers agreed that IL was strong compared } \\
\text { with when they agreed it was weak. Performance feedback } \\
\text { climate was higher when leaders and providers agreed that IL } \\
\text { was strong. }\end{array}$ \\
\hline $\begin{array}{l}\text { Brimhall } \\
\text { et al. } \\
2016[84]\end{array}$ & TfL & OC, IC & $\mathrm{N} / \mathrm{a}$ & Obs & $\mathrm{CMH}$ & $\mathrm{NI}$ & $\begin{array}{l}\text { Greater TfL indirectly influenced providers' perceptions of EBPs } \\
\text { as less burdensome through higher EmpCli and lower } \\
\text { DemoCli. }\end{array}$ \\
\hline $\begin{array}{l}\text { Bunger } \\
\text { et al., } \\
2019 \text { [65] }\end{array}$ & LB & OC & N/a & Obs & CW & । & $\begin{array}{l}\text { Leaders' activities influenced aspects of ImpCli including } \\
\text { conveying expectations, providing support, and rarely } \\
\text { rewarding implementation. Leaders conveyed expectations }\end{array}$ \\
\hline
\end{tabular}

\begin{tabular}{|c|c|c|c|c|c|c|}
\hline $\begin{array}{l}\text { First } \\
\text { author }\end{array}$ & $\begin{array}{l}\text { Leadership } \\
\text { type(s) }\end{array}$ & $\begin{array}{l}\text { Inner-context } \\
\text { outcomes }\end{array}$ & IS outcomes & Design & Setting & IS \\
\hline $\begin{array}{l}\text { Aarons } \\
2006 \text { [81] }\end{array}$ & TfL, TrL & IC & $\mathrm{N} / \mathrm{a}$ & Obs & $\mathrm{CMH}$ & $\mathrm{N}$ \\
\hline $\begin{array}{l}\text { Aarons } \\
\text { et al. } \\
2012 \text { [82] }\end{array}$ & $T f L$ & OC, IC; L & N/a & Exp & CW & I \\
\hline $\begin{array}{l}\text { Aarons } \\
\text { et al. } \\
2015 \text { [28] }\end{array}$ & TfL & OC & N/a & Obs & $\mathrm{MH}$ & N \\
\hline $\begin{array}{l}\text { Aarons } \\
\text { et al. } \\
2016 \text { [83] }\end{array}$ & $\begin{array}{l}\text { TfL, TrL; } \\
\text { PaL, LB }\end{array}$ & N/a & Sus & Obs & $\begin{array}{l}\mathrm{CMH}, \\
\mathrm{CW} \\
\text { Oth }\end{array}$ & Su \\
\hline $\begin{array}{l}\text { Aarons } \\
\text { et al. } \\
2017 \text { [45] }\end{array}$ & IL & OC & $\mathrm{N} / \mathrm{a}$ & Obs & $\mathrm{MH}$ & N \\
\hline $\begin{array}{l}\text { Brimhall } \\
\text { et al. } \\
2016 \text { [84] }\end{array}$ & TfL & OC, IC & N/a & Obs & $\mathrm{CMH}$ & N \\
\hline $\begin{array}{l}\text { Bunger } \\
\text { et al., } \\
2019 \text { [65] }\end{array}$ & LB & OC & N/a & Obs & CW & । \\
\hline
\end{tabular}

$\begin{aligned} & \text { Corrigan } \\ & \text { et al. }\end{aligned}$
2002 [85]

\begin{tabular}{|c|c|c|c|c|c|}
\hline $\begin{array}{l}\text { Fenwick } \\
\text { et al. } \\
2018 \text { [86] }\end{array}$ & TfL & $\mathrm{IC}, \mathrm{F}$ & N/a & Obs & $\mathrm{CMH}$ \\
\hline $\begin{array}{l}\text { Fleury } \\
\text { et al. } \\
2014 \text { [87] }\end{array}$ & LB & N/a & $\mathrm{Gl}$ & Obs & $\begin{array}{l}\text { MH, } \\
\text { Oth }\end{array}$ \\
\hline $\begin{array}{l}\text { Green } \\
\text { et al. } \\
2014 \text { [88] }\end{array}$ & TfL & OC & N/a & Obs & $\mathrm{CMH}$ \\
\hline $\begin{array}{l}\text { Guerrero } \\
\text { et al. } \\
2014 \text { [89] }\end{array}$ & LC & N/a & A & Obs & $\mathrm{AH}$ \\
\hline $\begin{array}{l}\text { Guerrero } \\
\text { et al. } \\
2020 \text { [15] }\end{array}$ & $\mathrm{IL}$ & IC & A & Obs & $\mathrm{AH}$ \\
\hline $\begin{array}{l}\text { Mancini } \\
\text { et al. } \\
2009 \text { [90] }\end{array}$ & LB & N/a & $\mathrm{Gl}$ & Obs & $\mathrm{H}, \mathrm{MH}$ \\
\hline
\end{tabular}

Greater TfL indirectly influenced providers' perceptions of EBPS as less burdensome through higher EmpCli and lower DemoCli.

Leaders' activities influenced aspects of ImpCli including conveying expectations, providing support, and rarely rewarding implementation. Leaders conveyed expectations through diffusing information, synthesizing information, mediating between agency strategy and day-to-day activities, and selling implementation. Leaders supported implementation through diffusing, synthesizing, and mediating. They conveyed rewards through diffusing.

$2002[85]$

$$
\text { veyed rewards through diffusing. }
$$

TfL was positively associated with transformational OrgCul. LfL and passive MBE were negatively associated with a TrC. TfL was positively associated with $\operatorname{TrC}$ based on leader report, but negatively associated with $\operatorname{TrC}$ based on provider report. Passive MBE was positively associated with $\operatorname{TrC}$ based on provider report. TfL was negatively associated with burnout among providers and leaders.

TfL and LMX positively predicted providers' attitudes toward feedback. LMX mediated the relation between TfL and providers' attitudes toward feedback.

$$
\text { providers' attitudes toward feedback. }
$$
Inaccessibility of leaders, leader turnover, and leaders' poor
communication were barriers to implementation.

Leaders' TfL positively predicted EmpCli.

Leaders' EBPA and readiness-for-change attributes positively predicted implementation of contingency management treatment. Leaders' openness towards EBPs positively predicted implementation of medication-assisted treatment.* IL was positively associated with provider's EBPA. IL did not
mediate the relation among top leaders' TfL and A.

Leaders' failure to empower staff, poor organizational skills, poor management of internal dynamics and workload, and turnover were barriers to high- $\mathrm{F}$ implementation. Leaders understanding of the model, effective management of team dynamics, holding staff accountable, advocating on behalf of 
Table 2 Summary of included studies $(N=21)$ (Continued)

\section{First Leadership Inner-context IS outcomes Design Setting IS phase Key leadership findings}

author type(s) outcomes

\begin{tabular}{|c|c|c|c|c|c|c|c|}
\hline & & & & & & & $\begin{array}{l}\text { provider teams, empowering staff, conveying a sense of mis- } \\
\text { sion to the provider team, and equitably distributing the work- } \\
\text { load facilitated implementation. }\end{array}$ \\
\hline $\begin{array}{l}\text { Moser } \\
\text { et al. } \\
2005[91]\end{array}$ & $L B, L C$ & N/a & $\mathrm{Gl}$ & Obs & $\mathrm{MH}$ & $P, I$ & $\begin{array}{l}\text { Leaders' turnover, lack of familiarity with the intervention, and } \\
\text { lack of investment in implementation were barriers to } \\
\text { implementation. Leader familiarity with the intervention } \\
\text { facilitated implementation. }\end{array}$ \\
\hline $\begin{array}{l}\text { Powell } \\
\text { et al. } \\
2017 \text { [92] }\end{array}$ & TfL, IL & IC & $\mathrm{N} / \mathrm{a}$ & Obs & $\mathrm{CMH}$ & I, Sus, NI & $\begin{array}{l}\text { TfL-idealized influence positively predicted providers' } \\
\text { knowledge of EBP. TfL-individual consideration negatively pre- } \\
\text { dicted providers' EBPA. Proactive IL positively predicted pro- } \\
\text { viders' EBPA. Perseverant IL negatively predicted providers' } \\
\text { EBPA. }\end{array}$ \\
\hline $\begin{array}{l}\text { Rapp } \\
\text { et al. } \\
2010[93]\end{array}$ & LB & N/a & $\mathrm{Gl}$ & Obs & $\mathrm{MH}$ & I & $\begin{array}{l}\text { Leader behaviors were the greatest barrier to implementation, } \\
\text { including: not setting expectations; only providing } \\
\text { consultation on service-delivery when challenges arose; lacking } \\
\text { prescriptions or structure to providers' practice; being overly } \\
\text { conflict-avoidant; lacking meaningful feedback for providers; } \\
\text { having only superficial knowledge of clinician practice; relying } \\
\text { on coaxing and persuasion with no consequences for poor } \\
\text { performance; poorly leading group supervision, which was } \\
\text { dominated by administrative tasks; lacking follow-through; } \\
\text { having competing responsibilities; lacking knowledge of EBP } \\
\text { skills and feeling inadequate at supervising practice. }\end{array}$ \\
\hline $\begin{array}{l}\text { Savill } \\
\text { et al. } \\
2018[94]\end{array}$ & LB & N/a & $\mathrm{Gl}$ & Obs & $\begin{array}{l}\mathrm{MH}, \\
\mathrm{SMH}\end{array}$ & I & $\begin{array}{l}\text { Leaders facilitated implementation by working to incorporate } \\
\text { EBP procedures into existing workflows (i.e., assessment } \\
\text { checklists and forms) and meeting regularly with senior } \\
\text { administrators and staff to monitor and troubleshoot } \\
\text { implementation difficulties. }\end{array}$ \\
\hline $\begin{array}{l}\text { Van Erp } \\
\text { et al. } \\
2007[95]\end{array}$ & $L B, L C$ & N/a & Gl & Obs & $\mathrm{MH}$ & । & $\begin{array}{l}\text { Lack of time for leaders to manage the intervention was a } \\
\text { barrier to implementation. Leaders' strong personal } \\
\text { commitment demonstrated by their dedication and } \\
\text { enthusiasm to implement the intervention facilitated } \\
\text { implementation. }\end{array}$ \\
\hline $\begin{array}{l}\text { Van Erp } \\
\text { et al. } \\
2009[96]\end{array}$ & LB & N/a & $\mathrm{Gl}$ & Obs & $\mathrm{H}, \mathrm{MH}$ & I & $\begin{array}{l}\text { Leaders' inability to administer the implementation process } \\
\text { and to realize necessary conditions for implementation were } \\
\text { barriers to implementation. Leader motivation facilitated } \\
\text { implementation. }\end{array}$ \\
\hline $\begin{array}{l}\text { Williams } \\
\text { et al. } \\
2020 \text { [97] }\end{array}$ & IL & OC & A & Quas & $\mathrm{CMH}$ & Mul & $\begin{array}{l}\text { Increases in IL had a significant indirect effect on increases in } \\
\text { clinicians' EBP use via improvement in EBP ImpCli. }\end{array}$ \\
\hline
\end{tabular}

$A$ adoption, $A H$ addiction health agencies, $C M H$ child mental health agencies, $C W$ child welfare, DemoCli demoralizing climate, $E B P$ evidence-based practice, $E B P A$ evidence-based practice attitudes, EmpCli empowering climate, Exp experimental, $F$ fidelity, $G l$, general implementation, $H$ hospital, $I$ implementation, $I C$ individual characteristics, IL implementation leadership, ImpCli implementation climate, InnCli innovation climate, $L$ leadership, $L B$ leader behaviors, $L C$ leader characteristics, $L f L$ laissez-faire leadership, $L M X$ leader-member exchange, $M B E$ management by exception, $M H$ mental health agencies, Mul multiple phases-unspecified, $N / A$ not applicable, NI no active implementation, NR not reported, Obs observational, OC organizational characteristics, OrgCli organizational climate, OrgCul organizational culture, Oth other, $P$ preparation, $P a L$ passive-avoidant leadership, Quas quasi-experimental, SMH school-based mental health, Sus sustainment, $T f L$ transformational leadership, $\operatorname{TrC}$ transactional culture, $\operatorname{TrL}$ transactional leadership

\section{Implementation outcomes}

Eleven studies (52\%) examined the relationship between leadership and an implementation outcome, including five studies (24\%) that examined a specific implementation outcome as defined by Proctor and colleagues [63]. These included adoption (14\%), fidelity (5\%), and sustainment (5\%). The remaining six studies (29\%) examined "general implementation," often encompassing multiple implementation outcomes.

\section{Relationships between leadership and inner-context outcomes}

A summary of the quantitative direct and indirect relationships between leadership and inner-context and implementation outcomes is included in Table 7. Results from the qualitative synthesis are listed in Table 8. Most studies (43\%) examined the relationship between general leadership and an inner-context outcome [45, 65, 81, 82, 84-86, 88, 92], and fewer studies examined the 
Table 3 Study characteristics ( $N=21)$

\section{Characteristics}

Setting ${ }^{a}$

Child welfare

Mental health agencies

Substance use agencies

Hospital

Other

Country

Canada

Netherlands

United States

Design

Observational

Quasi-/Experimental

Method

Quantitative

Qualitative

Mixed

Data source ${ }^{a}$

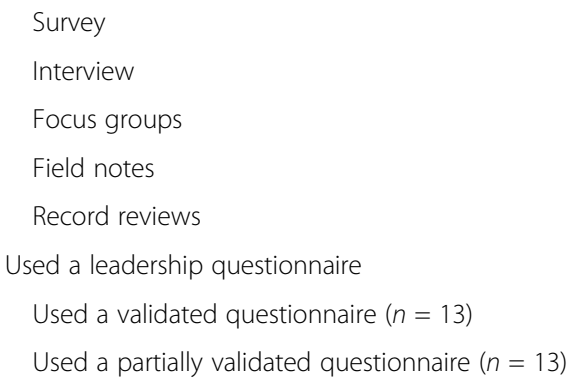

Interview

Focus groups

Field notes

Record reviews

Used a leadership questionnaire

Used a validated questionnaire $(n=13)$

Used a partially validated questionnaire $(n=13)$

$13(62 \%)$

$12(92 \%)$

Used an inner-context and/or implementation outcome questionnaire

Used a validated questionnaire $(n=15)$

$11(73 \%)$

Used a partially validated questionnaire $(n=15)$

$11(73 \%)$

Used an unvalidated questionnaire $(n=15)$

Implemented a clinical innovation

Phase of implementation ${ }^{a}$

Preparation

Implementation

Sustainment

Multiple phases (unspecified)

No active implementation

Not reported

Leadership examined $^{a}$

General leadership

Transformational leadership

Transactional leadership

Passive-avoidant leadership 
Table 3 Study characteristics $(N=21)$ (Continued)

\begin{tabular}{|c|c|}
\hline Characteristics & $N(\%)$ \\
\hline Strategic leadership & $12(57 \%)$ \\
\hline Implementation leadership & $11(52 \%)$ \\
\hline Middle-managers' roles in implementation ${ }^{\text {b }}$ & $6(29 \%)$ \\
\hline EBP champion ${ }^{\mathrm{b}}$ & $2(10 \%)$ \\
\hline Leader behaviors ${ }^{\mathrm{b}}$ (uncategorized) & $5(24 \%)$ \\
\hline Leader characteristics ${ }^{\mathrm{b}}$ (uncategorized) & $1(5 \%)$ \\
\hline Inner-context outcomes ${ }^{a}$ & $12(57 \%)$ \\
\hline Organizational characteristics ${ }^{a}$ & $8(38 \%)$ \\
\hline Organizational climate & $3(14 \%)$ \\
\hline Organizational culture & $2(10 \%)$ \\
\hline Implementation climate & $2(10 \%)$ \\
\hline Innovation climate & $1(5 \%)$ \\
\hline Individual characteristics ${ }^{a}$ & $8(38 \%)$ \\
\hline Attitudes towards EBP & $6(29 \%)$ \\
\hline Knowledge of EBP & $1(5 \%)$ \\
\hline Burnout & $1(5 \%)$ \\
\hline Implementation outcomes & $11(52 \%)$ \\
\hline Adoption & $3(14 \%)$ \\
\hline Fidelity & $1(5 \%)$ \\
\hline General implementation & $6(29 \%)$ \\
\hline Sustainment & $1(5 \%)$ \\
\hline
\end{tabular}

$N=21$ unless specified otherwise

${ }^{\text {a }}$ Responses are not mutually exclusive

${ }^{\mathrm{b}}$ The studies reporting these leadership roles and behaviors were all qualitative studies

relationship between strategic implementation leadership and an inner-context outcome (24\%) [15, 45, 65, 92, 97].

Individual characteristics The relationship between transformational leadership and providers' EBP attitudes was the most commonly examined relationship (24\%) across all outcomes. Four studies showed some evidence of a positive relationship between transformational leadership and providers' EBP attitudes [81, $82,84,86]$ and two supported a positive relationship between implementation leadership and EBP attitudes $[15,92]$. However, these studies also reported mixed leadership-attitude relationships. One study found transformational leadership positively predicted provider attitudes in the context of EBP implementation, but was unrelated to EBP attitudes among providers delivering services-as-usual [82]. Another study found

Table 4 Study level of analysis

\begin{tabular}{llll}
\hline Level & Leadership & Inner-context & Implementation \\
\hline Individual & $4(19 \%)$ & $9(43 \%)$ & $2(10 \%)$ \\
Team & $9(43 \%)$ & $4(19 \%)$ & $0(0 \%)$ \\
Organization & $8(38 \%)$ & $2(10 \%)$ & $9(43 \%)$ \\
\hline
\end{tabular}

positive, negative, and non-significant relationships between general and strategic leadership and EBP attitudes [92]. It is notable that this study included several theoretically correlated predictors in their model (e.g., general and strategic leadership), which raises the question of whether multicollinearity may explain the negative relationships between leadership and EBP attitudes. Individual consideration, one of four domains of transformational leadership involving attention to individual needs and feelings of employees, was negatively related to EBP attitudes. With regard to implementation leadership, proactive leadership, involving anticipating and addressing implementation challenges, was positively related to EBP attitudes. Another domain perseverant leadership, which is consistent, resolute, and responsive to EBP implementation, was negatively related to EBP attitudes [92].

Organizational characteristics While there were mixed relationships between leadership styles and organizational characteristics, three studies (14\%) supported a positive relationship between either transformational or implementation leadership and organizational climate $[45,84,99]$. Among the 
Table 5 Glossary of leadership constructs

\begin{tabular}{|c|c|}
\hline Term & Definition \\
\hline $\begin{array}{l}\text { First-level leadership [8-10, } \\
\text { 18] }\end{array}$ & $\begin{array}{l}\text { A process of intentional efforts, by an individual who directly supervises frontline employees who do not manage } \\
\text { others, to motivate, influence, and enable a person or group of people with the aim of impacting group or } \\
\text { organizational outcomes }\end{array}$ \\
\hline $\begin{array}{l}\text { Transformational leadership } \\
\text { [41] }\end{array}$ & Inspiring and motivating employees to perform beyond expectations \\
\hline Inspirational motivation & Possessing a shared vision and high expectations that inspire and motivate others \\
\hline Idealized influence & Embodying the values and behaviors to fulfill this vision \\
\hline Intellectual stimulation & Challenging others to rethink ways they perform their duties and soliciting their ideas \\
\hline $\begin{array}{l}\text { Individualized } \\
\text { consideration }\end{array}$ & Attending to the individual needs and feelings of employees \\
\hline Transactional leadership [41] & Relying on reinforcement and exchanges to promote performance on tasks that are part of one's role \\
\hline Contingent reward & Assigning and setting reward contingencies for fulfilling tasks \\
\hline $\begin{array}{l}\text { Management by } \\
\text { exception-active }\end{array}$ & Actively identifying and addressing employee mistakes or performance shortcomings \\
\hline $\begin{array}{l}\text { Passive-avoidant leadership } \\
\text { [42] }\end{array}$ & Avoiding making decisions and/or managing employees \\
\hline $\begin{array}{l}\text { Management by } \\
\text { exception-passive }\end{array}$ & Passively waiting for errors and issues and then addressing them \\
\hline Laissez-faire & Taking a "hands off" approach by altogether avoiding making decisions or managing employees \\
\hline $\begin{array}{l}\text { Implementation leadership } \\
\text { [20] }\end{array}$ & Leading in ways that are intended to promote the implementation of EBP \\
\hline Proactive & Anticipating and addressing implementation challenges \\
\hline Knowledgeable & Possessing a deep understanding of EBP implementation \\
\hline Perseverant & Being consistent, resolute, and responsive to EBP implementation \\
\hline Supportive & Supporting providers' EBP adoption and use \\
\hline EBP champion [64] & $\begin{array}{l}\text { Convincing others to accept the innovation through educating, advocating, building relationships and navigating } \\
\text { boundaries }\end{array}$ \\
\hline Effective organization & Coordinating employees and tasks to efficiently accomplish goals \\
\hline Managing team relationships & Promoting group cohesion by attending to social dynamics between employees \\
\hline Equitably distributing work & Dividing work tasks in a fair manner \\
\hline $\begin{array}{l}\text { Managing competing } \\
\text { priorities }\end{array}$ & Attending to implementation tasks among competing priorities and workloads \\
\hline Facilitating communication & Ensuring information is communicated across varying levels of an organization \\
\hline EBP buy-in & Agreeing with or supporting an EBP and its implementation \\
\hline
\end{tabular}

$E B P$ evidence-based practice

qualitative studies, one study (5\%) examined the relationship between leadership and an organizational characteristic [65]. High engagement in behaviors reflecting transformational leadership, implementation leadership, and middle managers' role in implementation supported implementation climate. Low engagement in implementation leadership, specifically supportive leadership, and the middle managers' role in implementation, hindered implementation climate.

Mediators of leadership and inner-context outcomes Three studies (14\%) examined the relationship between leadership and inner-context outcomes indirectly. The mediators examined included leader-provider relationship
[82, 86], innovation climate [82], and organizational climate [84]. The strength of the leader-follower relationship mediated the relationship between transformational leadership and EBP attitudes [86] and innovation climate [82]. Organizational climate mediated the relationship between transformational leadership and EBP attitudes [84].

\section{Relationships between leadership and implementation outcomes}

Eight studies (38\%) examined the relationship between strategic implementation leadership and an implementation outcome, the majority (75\%) of which were qualitative. The most studied relationships were the facilitating and hindering role of 
Table 6 Glossary of inner-context factors

\begin{tabular}{|c|c|}
\hline Term & Definition \\
\hline $\begin{array}{l}\text { Organizational } \\
\text { characteristics }\end{array}$ & Structures or processes that take place and/or exist in organizations that may influence implementation [29] \\
\hline $\begin{array}{l}\text { Implementation } \\
\text { climate }\end{array}$ & The extent to which organizational members perceive that innovation use is expected, supported, and rewarded [56] \\
\hline Innovation climate & The shared perception of the extent of an organizations' openness to new innovations [57] \\
\hline Organizational climate & $\begin{array}{l}\text { "The shared meaning organizational members attach to the events, policies, practices, and procedures they experience } \\
\text { and the behaviors they perceive being expected, rewarded and supported" [58] }\end{array}$ \\
\hline Empowering climate & Organizational members perceptions of fairness, perceived opportunities for growth and advancement, and role clarity [59] \\
\hline Demoralizing climate & Organizational members perceptions of depersonalization, emotional exhaustion, and role conflict [59] \\
\hline Organizational culture & The norms and shared behavioral expectations within an organization [60] \\
\hline Individual characteristics & Shared or unique characteristics of individuals (e.g., provider, supervisor, director) that influence implementation [29] \\
\hline Burnout & $\begin{array}{l}\text { A psychological syndrome characterized by emotional exhaustion, depersonalization, and reduced personal } \\
\text { accomplishment [61] }\end{array}$ \\
\hline Attitudes toward EBPs & $\begin{array}{l}\text { Provider perceptions of the appeal of EBPs, requirements to adopt EBPS, openness to innovation, and perceived } \\
\text { divergence between new and current practice [62] }\end{array}$ \\
\hline Knowledge of EBPs & Provider familiarity, awareness, or understanding of EBPs \\
\hline
\end{tabular}

$E B P$ evidence-based practice

implementation leadership (19\%) and middle managers' role in implementation (14\%) on general implementation. When leaders engaged in strategic implementation leadership, this facilitated general implementation and when leaders had an absence of strategic implementation leadership, this hindered general implementation. Two studies (10\%) quantitatively examined the relationship between implementation leadership and adoption. One found a positive association [97], while the other did not [15]. No studies quantitatively examined the relationship between strategic implementation leadership and sustainment.

Six studies (29\%) examined the relationship between general leadership and an implementation outcome [69, $83,87,93,95,96]$, the majority $(83 \%)$ of which were also qualitative. The relationship between general leadership and general implementation was most often studied. The presence of transformational leadership facilitated general implementation (10\%) $[95,96]$, while the presence of passive-avoidant leadership (10\%) [93, 96], and absence of transactional leadership (10\%) [87, 93] hindered general implementation. One study (5\%) quantitatively examined the relationship between general leadership and sustainment [83]. They found that transformational leadership was positively related to sustainment, transactional leadership was non-significantly related to sustainment, and passive-avoidant leadership was negatively associated with sustainment [83].

Mediators of leadership and implementation outcomes In the two studies $(10 \%)$ that examined the indirect relationship between leadership and an implementation outcome, one found that implementation climate mediated the relationship between implementation leadership and adoption [97], while the other did not find support for providers' EBP attitudes mediating the relationship between implementation leadership and adoption [15].

\section{Combined first-level and higher-level leadership}

The most notable differences between studies that combined perceptions of first-level leadership with higher leadership levels relative to studies that only examined first-level leadership were (1) study method, (2) styles of leadership examined, and (3) outcomes examined. Studies with mixed leadership tended to be qualitative (54\%), more often examined strategic implementation leadership $(77 \%)$, and more frequently examined a specific implementation outcome $(46 \%)$ or general implementation (46\%). Of note, $31 \%$ of these studies examined the relationship between leadership and sustainment, relative to only $5 \%$ in the first-level leadership only sample. These studies primarily provide additional support for the facilitating role of strategic implementation leadership, and to a lesser extent positively framed general leadership, in supporting implementation outcomes (see Table 12 in additional file 3 ).

\section{Conceptual overlap in leader behaviors}

Of all coded leader behaviors, $22 \%$ were categorized into more than one leadership style or theory. Among these overlapping codes, the majority came in two specific combinations. This included overlap in behaviors described in (1) the theories of implementation leadership and middle managers' role in implementation (58\%) and 
Table 7 Relationships between leadership and inner-context and implementation outcomes

\begin{tabular}{|c|c|c|c|c|c|c|}
\hline Leadership style & $N(\%)$ & Dir & Inner-context outcomes & $N(\%)$ & Dir & Implementation outcomes \\
\hline \multicolumn{7}{|l|}{ General leadership } \\
\hline \multirow[t]{6}{*}{ Transformational } & $1(5 \%)$ & - & Burnout [85] & $1(5 \%)$ & + & Sustainment [83] \\
\hline & $5(24 \%)$ & $+/-$ & EBP attitudes ${ }^{\mathrm{a}}[81,82,84,86,92]$ & & & \\
\hline & $1(5 \%)$ & $+/$ & EBP knowledge [92] & & & \\
\hline & $1(5 \%)$ & + & Innovation climate ${ }^{\mathrm{a}}[82]$ & & & \\
\hline & $2(10 \%)$ & + & Organizational climate $[84,88]$ & & & \\
\hline & $2(10 \%)$ & +- & Organizational culture $[85,98]$ & & & \\
\hline \multirow[t]{3}{*}{ Transactional } & $1(5 \%)$ & / & Burnout $^{\mathrm{c}}[85]$ & $1(5 \%)$ & / & Sustainment ${ }^{d}[83]$ \\
\hline & $1(5 \%)$ & + & EBP attitudes ${ }^{\mathrm{ab}}[81]$ & & & \\
\hline & $1(5 \%)$ & / & Organizational culture ${ }^{\mathrm{ac}}$ [85] & & & \\
\hline \multirow[t]{2}{*}{ Passive-avoidant } & $1(5 \%)$ & / & Burnout [85] & $1(5 \%)$ & - & Sustainment ${ }^{d}[83]$ \\
\hline & $1(5 \%)$ & -1 & Organizational culture ${ }^{a}[85]$ & & & \\
\hline \multicolumn{7}{|l|}{ Strategic leadership } \\
\hline \multirow[t]{4}{*}{ Implementation } & $2(10 \%)$ & $+/-$ & EBP Attitudes ${ }^{a}[15,92]$ & $2(10 \%)$ & +1 & Adoption $[15,97]$ \\
\hline & $1(5 \%)$ & / & EBP Knowledge [92] & & & \\
\hline & $1(5 \%)$ & + & Implementation Climate ${ }^{a}$ [97] & & & \\
\hline & $1(5 \%)$ & + & Organizational Climate ${ }^{\mathrm{a}}$ [45] & & & \\
\hline \multicolumn{7}{|l|}{ Leader characteristics } \\
\hline EBP attitudes & & & & $1(5 \%)$ & +1 & Adoption [89] \\
\hline Readiness-for-change & & & & $1(5 \%)$ & +1 & Adoption [89] \\
\hline
\end{tabular}

+ Significant positive association between constructs

- Significant negative association between constructs

/ Non-significant association between constructs

+/ Significant positive association and a non-significant association between constructs

-/ Significant negative association and a non-significant association between constructs

+- Significant positive and negative association between constructs

+/- Significant positive, significant negative, and non-significant between constructs

Statistical significance determined as $p<.05$. The direction of relationships can be mixed within a study if they report on subscales of a measure. We report only on total scores of a measure when available, otherwise, we report on subscale scores. Results reflect direct and indirect associations. When leader and provider scores were reported for the same measure, we report only the provider scores

${ }^{a}$ Reflects constructs that have been reframed to reflect the positive form of the construct (e.g., transformational leadership showing a negative association with demoralizing organizational climate is characterized as having a positive association with organizational climate)

${ }^{\mathrm{b}}$ Measurement of transactional leadership included contingent reward, active management-by-exception, passive management-by-exception, and laissez-faire

${ }^{c}$ Measurement of transactional leadership included contingent reward, active management-by-exception, and passive management-by-exception

dDomains comprising transactional leadership measurement were not defined

(2) transformational leadership and theory of middle managers' role in implementation (17\%).

The overlap in the theories of implementation leadership and middle managers' role in implementation occurred most (29\%) between two behaviors: (1) mediating between strategy and day-to-day activities ( $m e$ diating) and (2) proactive leadership (proactive). Notably, no implementation leadership codes overlapped with the middle manager role termed selling innovation implementation.

\section{Discussion}

This scoping review synthesized research examining the relationship between first-level leadership and innercontext and implementation outcomes in the context of behavioral health service delivery. Studies primarily relied on observational designs and were often crosssectional. Studies most often examined general forms of first-level leadership, namely transformational leadership. Strategic implementation leadership was also the focus of over half of studies. Nearly equal attention was given to inner-context and implementation outcomes, and inner-context outcomes centered on organizational and individual characteristics. The greatest concentration of studies examined first-level leadership in relation to providers' EBP attitudes and general implementation. Our synthesis revealed moderate conceptual overlap of leadership behaviors described in theoretical frameworks of strategic implementation leadership.

While the scope of the literature was broad, patterns emerged in the forms of leadership and outcomes studied across qualitative and quantitative 
Table 8 Barriers and facilitators of implementation outcomes and inner-context outcomes

\begin{tabular}{|c|c|c|c|c|}
\hline & Barriers & $N(\%)^{\mathrm{a}}$ & Facilitators & $N(\%)^{\mathrm{a}}$ \\
\hline \multicolumn{5}{|l|}{ Inner-context outcomes } \\
\hline \multirow[t]{5}{*}{ Implementation Climate } & Strategic leadership & $1(5 \%)$ & General leadership & $1(5 \%)$ \\
\hline & Low implementation leadership [65] & $1(5 \%)$ & High transformational leadership [65] & $1(5 \%)$ \\
\hline & Low middle managers' implementation role [65] & $1(5 \%)$ & Strategic leadership & $1(5 \%)$ \\
\hline & & & High implementation leadership [65] & $1(5 \%)$ \\
\hline & & & High middle managers' implementation roles [65] & $1(5 \%)$ \\
\hline \multicolumn{5}{|l|}{ Implementation outcomes } \\
\hline \multirow[t]{9}{*}{ Fidelity } & General leadership & $1(5 \%)$ & General leadership & $1(5 \%)$ \\
\hline & Low transformational leadership [90] & $1(5 \%)$ & High transformational leadership [90] & $1(5 \%)$ \\
\hline & Behaviors & $1(5 \%)$ & High transactional leadership [90] & $1(5 \%)$ \\
\hline & Inequitable workload distribution [90] & $1(5 \%)$ & Strategic leadership & $1(5 \%)$ \\
\hline & Poor organization [90] & $1(5 \%)$ & High implementation leadership [90] & $1(5 \%)$ \\
\hline & Poor management of team relations [90] & $1(5 \%)$ & High middle managers' implementation roles [90] & $1(5 \%)$ \\
\hline & & & Behaviors & $1(5 \%)$ \\
\hline & & & Equitable workload distribution [90] & $1(5 \%)$ \\
\hline & & & Good management of team relations [90] & $1(5 \%)$ \\
\hline \multirow[t]{11}{*}{ Implementation } & General leadership & $3(14 \%)$ & General leadership & $2(10 \%)$ \\
\hline & Low transactional leadership $[87,93]$ & $2(10 \%)$ & High transformational leadership $[95,96]$ & $2(10 \%)$ \\
\hline & High passive-avoidant leadership $[93,96]$ & $2(10 \%)$ & Strategic leadership & $3(10 \%)$ \\
\hline & Strategic leadership & $3(14 \%)$ & High implementation leadership $[91,94]$ & $2(10 \%)$ \\
\hline & Low implementation leadership $[91,93,96]$ & $3(14 \%)$ & $\begin{array}{l}\text { High middle managers' implementation roles } \\
{[91,94]}\end{array}$ & $2(10 \%)$ \\
\hline & $\begin{array}{l}\text { Low middle managers' implementation roles } \\
\text { [93] }\end{array}$ & $1(5 \%)$ & High EBP champion [95] & $1(5 \%)$ \\
\hline & Behaviors & $3(14 \%)$ & Characteristics & $1(5 \%)$ \\
\hline & $\begin{array}{l}\text { Poor management of competing priorities } \\
{[93,95]}\end{array}$ & $2(10 \%)$ & High EBP buy-in [68] & $1(5 \%)$ \\
\hline & Poor communication [87] & $1(5 \%)$ & & \\
\hline & Characteristics & $1(5 \%)$ & & \\
\hline & Low EBP buy-in [91] & $1(5 \%)$ & & \\
\hline \multirow[t]{4}{*}{ Sustainment } & & & Strategic leadership & $1(5 \%)$ \\
\hline & & & High implementation leadership [83] & $1(5 \%)$ \\
\hline & & & High middle managers' implementation roles [83] & $1(5 \%)$ \\
\hline & & & High EBP champion [83] & $1(5 \%)$ \\
\hline
\end{tabular}

Transactional leadership only refers to the domain contingent reward

investigations. Strategic leadership was more often the focus in qualitative studies, while quantitative studies commonly focused on general leadership. This likely reflects that quantitative measures of strategic implementation leadership have only recently been developed [46] while measures of general leadership have long been available [100]. Among outcomes studied, there was nearly equal representation of inner-context and implementation outcomes. However, over half of the literature informing our understanding of the relationship between first-level leadership and implementation measured general implementation, limiting our knowledge of the precise domains of implementation that are influenced by leadership. Further, of the five studies that measured a specific implementation outcome, three were self-reported. One notable exception was the use of independent observation to measure fidelity.

Few leadership-outcome relationships were examined across multiple studies; however, one exception was the focus on providers' EBP attitudes. Studies primarily found positive associations in the direct and indirect 
relationships between transformational leadership and providers' EBP attitudes, although there were exceptions. This finding aligns with a comprehensive synthesis of studies across occupational settings and leadership levels that support transformational leaderships' positive relation to employee attitudes [42]. In the implementation literature, theory suggests positive EBP attitudes are a necessary precursor to the EBP adoption decision [101], yet the evidence linking providers' attitudes and behavior is mixed. Studies conflict in their conclusions regarding providers' EBP attitudes and EBP implementationrelated behaviors, with some finding positive associations $[102,103]$ and others-including the most rigorous among these [104] -finding mixed or no associations [104-106]. What remains to be studied is whether the relationship between transformational leadership and providers' EBP attitudes has implications for providers' implementation behaviors. For instance, attitudes may modify the relationship between transformational leadership and adoption, such that transformational leadership may only predict adoption when providers have positive EBP attitudes.

Theory and research suggest general climates form a foundation for strategic climates, and these strategic climates function as a more proximal predictor of individual and organizational performance [58]. Ample evidence suggests that strategic climates mediate the relationship between leadership and employee performance $[107,108]$. Further, strategic leadership has shown a stronger effect on strategic climate than general high-performance leadership [107]. Theory within implementation also posits that leadership influences organizational characteristics, including culture and climate, which then shape provider adoption and sustainment of EBPs [4, 5, 109]. While the relationship between first-level leadership and various organizational characteristics was a primary focus of the reviewed studies, variability in the specific outcomes measured and inconsistent findings offer limited conclusions about the nature of this relationship. Studies revealed some consistent, yet sparse, support for general and strategic implementation leadership positively relating to general $[84,88$, 98] and strategic organizational climates [82, 97]. Studies demonstrated that general and strategic organizational climates mediated the relationship between positively framed leadership and other innercontext outcomes; however, only one study examined its mediating role on an implementation outcome. Williams and colleagues [97] provide initial evidence that the relationship between implementation leadership and EBP adoption is mediated by implementation climate. Extending from organizational theory and research, more evidence is needed to determine
(1) whether strategic implementation leadership is associated with better strategic implementation climate, (2) whether general leadership and general climate are necessary precursors for positive implementation leadership and implementation climate, and (3) the mediating role of organizational characteristics in the relationship between leadership and implementation outcomes.

Leadership has shown a positive relationship with employees' performance outcomes across industries [110]; however, the evidence linking leadership to implementation outcomes in behavioral health remains sparse. The qualitative evidence in this review indicates that firstlevel leadership, particularly strategic implementation leadership, facilitated general implementation. Yet it provides limited evidence regarding the influence of leadership behaviors on specific implementation outcomes. The studies involving mixed levels of leadership provide further support for the facilitating impact of strategic implementation leadership on implementation outcomes, although they preclude us from fully attributing that effect to first-level leadership. There were notably few studies that quantitatively examined the direct or indirect relationship between first-level leadership and implementation outcomes, limiting generalizations about these relationships. Findings suggest that implementation leadership and leader characteristics may support EBP adoption [89, 97], although additional research is needed as conflicting results exist [15]. Furthermore, measurement of EBP adoption has relied solely on provider report, dampening confidence in estimates of the leadership-adoption relationship [111].

\section{Broad strengths}

The reviewed studies had a number of strengths. Notably, studies drew from the organizational behavior literature to guide conceptualizations of leadership styles and organizational contextual factors that influence providers' EBP implementation. Use of validated and psychometrically sound measures of leadership and innercontext outcomes was a particular strength, especially given the lack of established implementation measures in the broader literature [112]. Studies tended to analyze perceptions of first-level leadership and implementation outcomes at the team and organization level, respectively. This aligns with calls for leadership research to move beyond studying how leadership affects individuallevel processes [17]. Nonetheless, they also had a number of limitations that inform our future recommendations.

\section{Recommendation 1: improve methodological quality}

Measurement and study design issues threaten progress in understanding how first-level leadership relates to 
implementation. Studies relied heavily on self-report measures, often by a single reporter. Most of the studies that quantitatively measured an implementation outcome were based on self-report. These measurement issues have produced overestimated parameters for leadership-outcome relationships [42, 111], and may contribute to inconsistent or conflicting results. While self-report may be most appropriate for many innercontext outcomes conceptualized as latent constructs, implementation outcomes are often directly observable. To address common method variance, studies should rely on multiple respondents for self-reported outcomes, such as measuring aggregate perceptions of organizational characteristics from all available employees, not only those reporting on leadership. When common methods are unavoidable, studies should follow procedural and statistical remedies to reduce bias (see Podsakoff and colleagues [113]). Studies should use direct observation of practice and record review to measure implementation outcomes such as adoption, fidelity and sustainment.

Two-thirds of studies that examined the indirect relationship between leadership and outcomes did so using a cross-sectional observational design, likely producing biased estimates [42]. While comprehensive recommendations for mediation analysis are described elsewhere $[114,115]$, we highlight those that are most feasible and relevant. Studies should include (1) more than two assessment points to establish temporal precedence [115]; (2) variables measured at all time points to assess for the reciprocity of mediating effects (e.g., changes in implementation climate lead to changes in EBP adoption and not vice versa) [114]; and (3) measurement should occur when changes in the mediator are expected to cause changes in the inner-context or implementation outcome.

\section{Recommendation 2: address conceptual overlap in first- level leadership}

There have been calls for clarification of the converging and unique contributions of the theory of implementation leadership [46] and the theory of middle managers' role in implementation [47], noting similarities in the behaviors described [97]. Our synthesis supports the claim that these theories are partially overlapping and suggests that middle managers' implementation role of mediating between strategy and day-to-day activities converges with proactive leadership. These concepts overlap in their focus on removing implementation obstacles, addressing barriers to implementation, and supporting a strategy or plan for implementation. Our findings also suggest that these theories diverge with regard to middle managers' implementation role of selling the innovation, a role defined as "presenting, convincing, and encouraging stakeholders to participate in implementation of an innovation" [49] (p. 9). In our synthesis, selling the innovation emerged as one of the most common ways that first-level leadership facilitated implementation, suggesting that it is a unique and important leadership behavior. Future research should examine the consistent and divergent aspects of these theories. For instance, template analysis could be used to explore this overlap, as was recently conducted to examine compatibility of the Ottawa Model of Implementation Leadership and the Implementation Leadership Scale [116].

\section{Recommendation 3: greater focus on implementation outcomes}

One notable gap was the limited focus on specific implementation outcomes. While our qualitative synthesis suggests strategic implementation leadership and general leadership facilitates general implementation, the imprecise measurement limits our understanding of which outcomes they impact. Given their direct role in guiding treatment delivery and close relationship with providers [117], first-level leadership likely influences implementation outcomes that reflect providers' EBP perceptions (e.g., acceptability) and their behaviors (e.g., adoption). Studies failed to examine implementation outcomes reflecting provider perceptions; however, these are likely related to the behavioral implementation outcomes [63]. For instance, the middle managers' implementation roles mediating and selling may improve providers' perceptions of EBP appropriateness, which may increase adoption. The literature would benefit from an increased focus on a broader set of implementation outcomes.

\section{Recommendation 4: examine how first-level leadership shapes team-level outcomes}

The broader leadership literature has been limited by a dearth of studies examining how leadership impacts team-level outcomes [17]. In behavioral health, frontline employees often interact with first-level leaders as a team and deliver services as a team. For instance, a majority of providers in community mental health receive group-based supervision [117] and providers often use a team-approach to treatment delivery such group-based treatments [118] and team approaches for treating highrisk clients and severe mental illness [35, 119]. Future research should explore whether first-level leadership affects inner-context and implementation outcomes at the team-level.

\section{Recommendation 5: explore conditions under which leadership shapes implementation}

Implementation is complex and highly context-dependent [120]. The inconsistencies in some leadership-outcome relationships may, in part, result from contextual factors that moderate these relationships. Studies of moderators 
were rare, yet two studies found that the influence of leadership on inner-context outcomes depended on whether an organization was actively implementing an innovation [82] and the degree of organizational stress [70]. The limited focus on moderators may miss characteristics of the organization, leader, provider, and EBP on which leadership-outcome relationship may depend. Leader characteristics, including EBP attitudes [89], EBP perceptions [71], and EBP buy-in [72, 91] that were related to implementation outcomes may moderate the relationship between leadership and implementation outcomes. The leadership literature points to possible provider-level moderators, such as self-efficacy [121], innovativeness [122], job tenure [123], and congruence of work with one's values [123]. Future studies should explore moderators of leadership and inner-context and implementation outcomes. This will begin to address the question of, under what circumstances does first-level leadership impact inner-context and implementation outcomes?

\section{Limitations}

Findings should be considered within the context of several limitations. First, we only included peer-reviewed empirical studies. As such, we excluded non-peerreviewed studies and study protocols. Recent protocols show promising methods that will likely advance our understanding of first-level leadership and implementation $[20,124]$. Further, while the inclusion of diverse study designs was most appropriate to address our aims, this precluded determinations about the validity of findings or reasons for discrepant findings in our sample. While search terms were developed to capture studies from diverse settings, most studies were conducted in the USA which may introduce bias.

\section{Conclusions}

This review offers a synthesis of the current state of the literature examining how first-level leadership shapes implementation. The findings suggest that the impact of leadership in shaping inner-context outcomes, most notably providers' EBP attitudes and the organizational context, has been a primary focus in quantitative analyses. In contrast, the facilitating and hindering role of strategic implementation leadership on general implementation have comprised much of the qualitative examinations. Our synthesis documents an important conceptual overlap in the theories of implementation leadership and middle managers' role in implementation that should be disentangled, as these forms of strategic leadership appear to hold promise in supporting EBP implementation. We identified gaps in the literature and provide recommendations to advance our understanding of the relationship between firstlevel leadership and inner-context and implementation outcomes.

\section{Abbreviations}

A: Adoption; AH: Addiction health agencies; Bar: Barriers of implementation; $\mathrm{CMH}$ : Child mental health agencies; CW: Child welfare; DemoCli: Demoralizing climate; EBP: Evidence-based practice; EBPA: Evidence-based practice attitudes; EmpCli: Empowering climate; EPIS: Exploration, Preparation, Implementation, and Sustainment; Exp: Experimental; F: Fidelity; Fac: Facilitators of implementation; Gl: General implementation; H: Hospital; I: Implementation; IC: Individual characteristics; IPP: Implementation policies and practices; IL: Implementation leadership; ImpCli: Implementation climate; InnCli: Innovation climate; L: Leadership; LB: Leader behaviors; LC: Leader characteristics; LfL: Laissez-faire leadership; LMX: Leader-member exchange; MBE: Management by exception; $\mathrm{MH}$ : Mental health agencies; Mul: Multiple phases-unspecified; N/A: Not applicable; NI: No active implementation; NR: Not reported; Obs: Observational; OC: Organizational characteristics; OrgCli: Organizational climate; OrgCul: Organizational culture; OSP: Organizational staffing processes; Oth: Other; P: Preparation; PaL: Passive-avoidant leadership; PRIS MA-ScR: Preferred Reporting Items for Systematic Reviews and Meta-Analyses Extension for Scoping Reviews; Quas: Quasi-experimental; SMH: School-based mental health; Sus: Sustainment; TfL: Transformational leadership;

TrC: Transactional culture; TrL: Transactional leadership; VA: Veteran's Affairs Integrated Service Networks

\section{Supplementary Information}

The online version contains supplementary material available at https://doi. org/10.1186/s13012-021-01104-4.

Additional File 1. Preferred Reporting Items for Systematic reviews and Meta-Analyses extension for Scoping Reviews (PRISMA-SCR) Checklist.

Additional File 2. Literature Search Terms.

Additional File 3. Mixed-level Leadership Results.

\section{Acknowledgements}

We would like to acknowledge Jill Locke, Ph.D., Michael Pullmann, Ph.D., Wendy Stone, Ph.D., and Lori Zoellner, Ph.D. for their feedback on study design and manuscript drafts.

\section{Authors' contributions}

RM conceived of the review and engaged in all aspects of study design and execution and drafted portions of the manuscript. NT provided guidance on methodology and design, assisted with articles screening and data extraction, and drafted portions of the manuscript and gave feedback on manuscript drafts. GW provided guidance on methodology and design, assisted with articles screening and extraction, and gave feedback on manuscript drafts. PM provided guidance on data synthesis and provided feedback on manuscript drafts. AK and GTJ assisted with article screening and gave feedback on manuscript drafts. SD provided guidance on study design and provided feedback on manuscript drafts. The author(s) read and approved the final manuscript.

\section{Funding}

This work was supported by a grant from the National Institute of Mental Health (S.D. R01 MH112633-02S1). Funding supported the first author's time dedicated to this manuscript.

\section{Availability of data and materials}

All articles included in this scoping review are publicly available. The datasets used and/or analyzed during the current study are available from the corresponding author on reasonable request.

\section{Declarations}

Ethics approval and consent Not applicable.

Consent for publication Not applicable. 


\section{Competing interests}

The authors declare that they have no competing interests.

Received: 20 August 2020 Accepted: 19 March 2021 Published online: 06 July 2021

\section{References}

1. Weisz JR, Ng MY, Bearman SK. Odd couple? Reenvisioning the relation between science and practice in the dissemination-implementation era. Assoc Psychol Sci. 2016;2(1):58-74.

2. Miller WR, Sorensen JL, Selzer JA, Brigham GS. Disseminating evidencebased practices in substance abuse treatment: a review with suggestions. J Subst Abus Treat. 2006;31(1):25-39 https://doi.org/10.1016/j.jsat.2006.03.005.

3. Bruns EJ, Ph D, Kerns SEU, Ph D, Pullmann MD, Ph D, et al. Research, data, and evidence-based treatment use in state behavioral health systems, 2001 - 2012. Psychiatr Serv. 2015;67(5):496-503 https://doi.org/10.1176/appi.ps.2 01500014.

4. Aarons GA, Hurlburt MS, Horwitz S. Advancing a conceptual model of evidence-based practice implementation in public service sectors. Adm Policy Ment Health Ment Health Serv Res. 2011;38(1):4-23 https://doi.org/1 0.1007/s10488-010-0327-7.

5. Damschroder LJ, Aron DC, Keith RE, Kirsh SR, Alexander J. a, Lowery JC. Fostering implementation of health services research findings into practice: a consolidated framework for advancing implementation science. Implement Sci. 2009;4(50):40-55.

6. Fixen DL, Naoom SF, Blase KB, Friedman RM, Wallace F. Implementation research: a synthesis of the literature: Louis de la Parte Florida Mental Health Institute; 2005.

7. Flottorp SA, Oxman AD, Krause J, Musila NR, Wensing M, Godycki-Cwirko M, et al. A checklist for identifying determinants of practice: a systematic review and synthesis of frameworks and taxonomies of factors that prevent or enable improvements in healthcare professional practice. Implement Sci. 2013:8(1):35 https://doi.org/10.1186/1748-5908-8-35.

8. Reichenpfader U, Carlfjord S, Nilsen P. Leadership in evidence-based practice: a systematic review. Leadersh Health Serv. 2015;28(4):298-316 https://doi.org/10.1108/LHS-08-2014-0061.

9. Gifford W, Davies B, Edwards N, Griffin P, Lybanon V. Managerial leadership for nurses' use of research evidence: an integrative review of the literature. Worldviews Evid Based Nurs. 2007;4(3):126-45 https://doi.org/10.1111/j.17416787.2007.00095.x

10. Ovretveit J. Improvement leaders: what do they and should they do? A summary of a review of research. Qual Saf Heal Care. 2010;19(6):490.

11. Gill R. Theory and Practice of Leadership. London: Sage Publications; 2012.

12. Yukl G. Leadership in Organizations. 6th ed. Upper Saddle River: Pearson/ Prentice Hall; 2006.

13. House RJ, Hanges PJ, Javidan M, Dorfman PW, Gupta V. Culture, leadership, and organizations: the GLOBE study of 62 societies: Sage publications; 2004.

14. Klein KJ, Sorra JS. The challenge of innovation implementation. Acad Manag Rev. 1996;21(4):1055-80 https://doi.org/10.5465/amr.1996.9704071863.

15. Guerrero EG, Frimpong J, Kong Y, Fenwick KM, Aarons GA, Kong Y. Advancing theory on the multilevel role of leadership in the implementation of evidence-based health care practices. Health Care Manag Rev. 2020;45(2):151-61 https://doi.org/10.1097/HMR. 0000000000000213.

16. Aarons GA, Ehrhart MG, Farahnak LR, Sklar M. Aligning leadership across systems and organizations to develop a strategic climate for evidencebased practice implementation. Annu Rev Public Health. 2014;35(1):255-74 https://doi.org/10.1146/annurev-publhealth-032013-182447.

17. Dechurch LA, Hiller NJ, Murase T, Doty D, Salas E. Leadership across levels: levels of leaders and their levels of impact. Leadersh Q. 2010;21(6):1069-85 https://doi.org/10.1016/j.leaqua.2010.10.009.

18. Priestland A, Hanig R. Developing first-level leaders. Harv Bus Rev. 2005; 83(6):112-20.

19. Jacobs TO, McGee ML. Competitive advantage: conceptual imperatives for executives. In: Zaccaro SJ, Klimoski RJ, editors. The nature of organizational leadership: Understanding the performance imperatives confronting today's leaders. San Francisco: Jossey-Bass; 2001. p. 42-78.

20. Aarons GA, Ehrhart MG, Moullin JC, Torres EM, Green AE. Testing the leadership and organizational change for implementation (LOCI) intervention in substance abuse treatment: A cluster randomized trial study protocol. Implement Sci. 2017;12(1):1-11.
21. McMillen JC, Raffol M. Characterizing the quality workforce in private U.S. child and family behavioral health agencies. Adm Policy Ment Health Ment Health Serv Res. 2016;43(5):750-9 https://doi.org/10.1007/s10488-015-0667-4.

22. Broome KM, Knight DK, Edwards JR, Flynn PM. Leadership, burnout, and job satisfaction in outpatient drug-free treatment programs. J Subst Abus Treat. 2009;37(2):160-70 https://doi.org/10.1016/j.jsat.2008.12.002.

23. Sonnino R. Health care leadership development and training: progress and pitfalls. J Healthc Leadersh. 2016:8:19-29 https://doi.org/10.2147/JHL.S68068.

24. Richman RC, Morahan PS, Cohen DW, McDade SA. Advancing women and closing the leadership gap: the executive leadership in academic medicine (ELAM) program experience. J Womens Heal Gender Based Med. 2001;10(3): 271-7 https://doi.org/10.1089/152460901300140022.

25. Rosenman ED, Shandro JR, Ilgen JS, Harper AL, Fernandez R. Leadership training in health care action teams: a systematic review. Acad Med. 2014; 89(9):1295-306 https://doi.org/10.1097/ACM.0000000000000413.

26. Sonnino RE. Professional development and leadership training opportunities for healthcare professionals. Am J Surg. 2013;206(5):727-31 https://doi.org/1 0.1016/j.amjsurg.2013.07.004.

27. Proctor E, Ramsey AT, Brown MT, Malone S, Hooley C, McKay V. Training in implementation practice leadership (TRIPLE): evaluation of a novel practice change strategy in behavioral health organizations. Implement Sci. 2019; 14(1):1-11.

28. Aarons GA, Ehrhart MG, Farahnak LR, Hurlburt MS. Leadership and organizational change for implementation (LOCI): a randomized mixed method pilot study of a leadership and organization development intervention for evidence-based practice implementation. Implement Sci. 2015;10(1):11 https://doi.org/10.1186/s13012-014-0192-y.

29. Moullin JC, Dickson KS, Stadnick NA, Rabin B, Aarons GA. Systematic review of the Exploration, preparation, implementation, sustainment (EPIS) framework. Implement Sci. 2019;14(1):1-16 https://doi.org/10.1186/s13012018-0842-6

30. Ansmann L, Pfaff H. Providers and patients caught between standardization and individualization: individualized standardization as a solution. Int J Health Policy Manag. 2018:7:349-52.

31. Miller WR, Rollnick $\mathrm{S}$. The effectiveness and ineffectiveness of complex behavioral interventions: Impact of treatment fidelity. Contemp Clin Trials. 2014;37(2):234-41 https://doi.org/10.1016/j.cct.2014.01.005.

32. Mchugh RK, Barlow DH, Kathryn R. The dissemination and implementation of evidence- based psychological treatments. 2010;65(2):73-84.

33. Wiltsey Stirman S, Gutner AC, Crits-Christoph P, Edmunds J, Evans AC, Beidas RS. Relationships between clinician-level attributes and fidelityconsistent and fidelity-inconsistent modifications to an evidence-based psychotherapy. Implement Sci. 2015;10(1):115.

34. Schoenwald SK, Garland AF. A review of treatment adherence measurement methods. Psychol Assess. 2013;25(1):1-20.

35. Linehan M. Cognitive-behavioral treatment of borderline personality disorder. New York: Guilford Press; 1993. p. 1-558.

36. Miller WR, Rollnick S. Motivational interviewing: helping people change: Guilford Press; 2012. p. 1-471.

37. Lock J, le Grange D. Family-based treatment of eating disorders. Int J Eat Disord. 2005:37:S64-7.

38. Yukl G, Gordon A, Taber T. Taxonomy of leadership behavior : half century of behavior research. J Leadersh Organ Behav. 2002;9(1):15-32 https://doi. org/10.1177/107179190200900102.

39. Bass BM. Leadership and performance beyond expectations. New York: Free Press; 1985.

40. Avolio BJ, Yammarino FJ. Introduction to, and overview of, transformational and charismatic leadership. In: Avolio BJ, Yammarino FJ, editors. Transformational and charismatic leadership: the road ahead. St Louis: Elsevier; 2002. p. xxvii-xxxiii.

41. Burns JM. Leadership. New York: Harper \& Row; 1978

42. Judge TA, Piccol RF. Transformational and transactional leadership: a metaanalytic test of their relative validity. J Appl Psychol. 2004;89(5):755-68 https://doi.org/10.1037/0021-9010.89.5.755.

43. Bass BM, Avolio BJ. Improving organizational effectiveness through transformational leadership. Thousand Oaks: Sage Publications; 1994.

44. Gifford WA, Squires JE, Angus DE, Ashley LA, Brosseau L, Craik JM, et al. Managerial leadership for research use in nursing and allied health care professions: A systematic review. Implement Sci. 2018;13(1):1-23.

45. Aarons GA, Ehrhart MG, Torres EM, Finn NK, et al. The humble leader: Association of discrepancies in leader and follower ratings of 
implementation leadership with organizational climate in mental health. Psychiatr Serv. 2017;68(2):115-22 https://doi.org/10.1176/appi.ps.201600062.

46. Aarons GA, Ehrhart MG, Farahnak LR. Implementation leadership scale (ILS): Development of a brief measure of unit level implementation leadership. Implement Sci. 2014;9(1):1-10.

47. Birken SA, Lee S-YD, Weiner BJ. Uncovering middle managers' role in healthcare innovation implementation. Implement Sci. 2012;7(28):1-12.

48. Birken SA, Dimartino LD, Kirk MA, Lee SD, McClelland M, Albert NM. Elaborating on theory with middle managers' experience implementing healthcare innovations in practice. Implement Sci. 2015;11(1):2 https://doi. org/10.1186/s13012-015-0362-6.

49. Birken SA, Clary A, Tabriz AA, Turner K, Meza R, Zizzi A, et al. Middle managers ' role in implementing evidence-based practices in healthcare : a systematic review. Implement Sci. 2018;13(1):1-14.

50. Birken SA, Lee S-YD, Weiner BJ, Chin MH, Schaefer CT. Improving the effectiveness of health care innovation implementation: Middle managers as change agents. Med Care Res Rev. 2013;70(1):29-45 https://doi.org/10.11 77/1077558712457427.

51. Williams NJ, Ehrhart MG, Aarons GA, Marcus SC, Beidas RS. Linking molar organizational climate and strategic implementation climate to clinicians' use of evidence-based psychotherapy techniques: cross-sectional and lagged analyses from a 2-year observational study. Implement Sci. 2018; 13(85):1-13.

52. Williams NJ, Frank HE, Frederick L, Beidas RS, Mandell DS, Aarons GA, et al. Organizational culture and climate profiles: relationships with fidelity to three evidence-based practices for autism in elementary schools. Implement Sci. 2019;14(15):1-14

53. Peters MDJ, Godfrey CM, Khalil H, Mclnerney P, Parker D, Soares CB. Guidance for conducting systematic scoping reviews. Int J Evid Based Healthc. 2015;13(3):141-6 https://doi.org/10.1097/XEB.0000000000000050.

54. Arksey H, O'Malley L. Scoping studies: Towards a methodological framework. Int J Soc Res Methodol Theory Pract. 2005;8(1):19-32 https://doi. org/10.1080/1364557032000119616.

55. Anderson S, Allen P, Peckham S, Goodwin N. Asking the right questions: scoping studies in the commissioning of research on the organisation and delivery of health services. Heal Res Policy Syst. 2008;6:1-12.

56. Weiner BJ, Belden CM, Bergmire DM, Johnston M. The meaning and measurement of implementation climate. Implement Sci. 2011;6(1):78 https://doi.org/10.1186/1748-5908-6-78.

57. Anderson NR, West MA. Measuring climate for work group innovation: development and validation of the team climate inventory. J Organ Behav. 1998;19(3):235-58 https://doi.org/10.1002/(SICI)1099-1379(199805)19:3<23 5::AID-JOB837>3.0.CO;2-C.

58. Ehrhart MG, Schneider B, Macey WH. Organizational climate and culture: an introduction to theory, research, and practice. New York: Routledge; 2014.

59. Mowday RT, Porter LW, Steers RM. Employee-organization linkages: the psychology of commitment, absenteeism, and turnover, vol. 1. 1st ed. New York: Academic press; 1982. p. 264.

60. Glisson C, James LR. The cross-level effects of culture and climate in human service teams. J Organ Behav Int J Ind Occup Organ Psychol Behav. 2002; 23(6):767-94.

61. Maslach C, Jackson SE, Leiter MP, Schaufeli WB, Schwab RL. Maslach burnout inventory. Palo Alto: Consulting psychologists press; 1986.

62. Aarons GA. Mental health provider attitudes toward adoption of evidence-based practice: the evidence-based practice attitude scale (EBPAS). Ment Health Serv Res. 2004;6(2):61-74 https://doi.org/10.1023/B:MHSR.0000024351.12294.65.

63. Proctor $E$, Silmere H, Raghavan R, Hovmand P, Aarons GA, Bunger A, et al. Outcomes for implementation research: conceptual distinctions, measurement challenges, and research agenda. Adm Policy Ment Health Ment Health Serv Res. 2011;38(2):65-76 https://doi.org/10.1007/s10488-010-0319-7.

64. Soo S, Berta W, Baker GR. Role of champions in the practice change. Healthc Q. 2009;12(sp):123-8 https://doi.org/10.12927/hcq.2009.20979.

65. Bunger AC, Birken SA, Hoffman JA, MacDowell H, Choy-Brown M, Magier E. Elucidating the influence of supervisors' roles on implementation climate. Implement Sci. 2019;14(1):1-12

66. Walumbwa FO, Avolio BJ, Gardner WL, Wernsing TS, Peterson SJ. Authentic leadership: development and validation of a theory-based measure. J Manag. 2008;34(1):89-126.

67. Graen GB, Mary U. Development of leader-member exchange (LMX) theory of leadership over 25 years: Applying a multi-level multi-domain perspective. Leadersh Q. 1995;6(2):219-47 https://doi.org/10.1016/1048-9843(95)90036-5.
68. Brooks H, Pilgrim D, Rogers A. Innovation in mental health services: what are the key components of success? Implement Sci. 2011;6(1):120 https:// doi.org/10.1186/1748-5908-6-120.

69. Byron G, Ziedonis DM, MCGrath C, Frazier JA, DeTorrijos F, Fulwiler C. Implementation of mindfulness training for mental health staff: Organizational context and stakeholder perspectives. Mindfulness ( $N$ Y). 2015;6(4):861-72 https://doi.org/10.1007/s12671-014-0330-2.

70. Aarons GA, Sommerfeld DH, Willging CE. The soft underbelly of system change: the role of leadership and organizational climate in turnover during statewide behavioral health reform. Psychol Serv. 2011;8(4):269-81 https:// doi.org/10.1037/a0026196.

71. Rodriguez A, Lau AS, Wright B, Regan J, Brookman-Frazee L. Mixed-method analysis of program leader perspectives on the sustainment of multiple child evidence-based practices in a system-driven implementation. Implement Sci. 2018;13:1.

72. Whitley R, Gingerich S, Lutz WJ, Mueser KT. Implementing the illness management and recovery program in community mental health settings: facilitators and barriers. Psychiatr Serv. 2009;60(2):202-9 https://doi.org/10.11 76/ps.2009.60.2.202.

73. Einfeld SL, Beard J, Tobin M, Buss R, Dudley M, Clarke AR, et al. Evidencebased practice for young people who self harm: can it be sustained and does it improve outcomes? Aust Health Rev. 2002;25(4):178-88 https://doi. org/10.1071/AH020178.

74. Nadeem E, Saldana L, Chapman J, Schaper H. A mixed methods study of the stages of implementation for an evidence-based trauma intervention in schools. Behav Ther. 2018;49(4):509-24 https://doi.org/10.1016/j.beth.201 7.12.004.

75. Fearing G, Barwick M, Kimber M. Clinical transformation: manager's perspectives on implementation of evidence-based practice. Adm Policy Ment Health Ment Health Serv Res. 2014;41(4):455-68 https://doi.org/10.1 007/s10488-013-0481-9.

76. Stein BD, Celedonia KL, Kogan JN, Swartz HA, Frank E. Facilitators and barriers associated with implementation of evidence-based psychotherapy in community settings. Psychiatr Serv. 2013;64(12):1263-6 https://doi.org/1 0.1176/appi.ps.201200508.

77. Xanidis N, Gumley A. Exploring the implementation of cognitive behaviour therapy for psychosis using the Normalization Process Theory framework. Psychol Psychother. 2020;93(2):241-57.

78. Guerrero EG, Padwa H, Fenwick KM, Harris LM, Aarons GA. Identifying and ranking implicit leadership strategies to promote evidence-based practice implementation in addiction health services. Implement Sci. 2016;11(69):113.

79. Sommerfeld DH, Granholm E, Holden J, Seijo C, Rapoport CS, Mueser KT, et al. Concept mapping study of stakeholder perceptions of implementation of cognitive-behavioral social skills training on assertive community treatment teams. Psychol Serv. 2021;18(1):33-41.

80. Ford $\mathrm{JH}$, Krahn D, Wise M, Oliver KA. Measuring sustainability within the veterans administration mental health system redesign initiative. Qual Manag Health Care. 2011;20(4):263-79 https://doi.org/10.1097/QMH.0b013 e3182314b20.

81. Aarons GA. Transformational and transactional leadership: association with attitudes toward evidence-based practice. Psychiatr Serv. 2006;57(8):1162-9 https://doi.org/10.1176/ps.2006.57.8.1162.

82. Aarons GA, Sommerfeld D. Leadership, innovation climate, and attitudes toward evidence-based practice during a statewide implementation. J Am Acad Child Adolesc Psychiatry. 2012;51(4):423-31 https://doi.org/10.1016/j. jaac.2012.01.018

83. Aarons GA, Green AE, Trott E, Willging CE, Torres EM, Ehrhart MG, et al. The roles of system and organizational leadership in system-wide evidencebased intervention sustainment: A mixed-method study. Adm Policy Ment Health Ment Health Serv Res. 2016;43(6):991-1008 https://doi.org/10.1007/s1 0488-016-0751-4.

84. Brimhall KC, Fenwick KM, Farahnak LR, Hurlburt MS, Roesch SC, Aarons GA. Leadership, organizational climate, and perceived burden of evidence-based practice in mental health services. Adm Policy Ment Health Ment Health Serv Res. 2016:43(5):629-39 https://doi.org/10.1007/s10488-015-0670-9.

85. Corrigan PW, Diwan S, Campion J, Rashid F. Transformational leadership and the mental health team. Admin Pol Ment Health. 2002;30(2):97-108 https:// doi.org/10.1023/A:1022569617123.

86. Fenwick KM, Brimhall KC, Hurlburt M, Aarons GA. Who wants feedback? Effects of transformational leadership and leader-member exchange on 
mental health practitioners' attitudes toward feedback. Psychiatr Serv. 2018; 70(1):11-8 https://doi.org/10.1176/appi.ps.201800164

87. Fleury M-J, Grenier $G$, Vallée C. Evaluation of the implementation of the Montreal at home/chez soi project. BMC Health Serv Res. 2014;14(1).

88. Green AE, Albanese BJ, Cafri G, Aarons GA. Leadership, organizational climate, and working alliance in a children's mental health service system. Community Ment Health J. 2014;50(7):771-7, 777 https://doi.org/10.1007/s 0597-013-9668-5.

89. Guerrero EG, He A, Kim A, Aarons GA. Organizational implementation of evidence-based substance abuse treatment in racial and ethnic minority communities. Adm Policy Ment Health Ment Health Serv Res. 2014;41(6): 737-49 https://doi.org/10.1007/s10488-013-0515-3.

90. Mancini AD, Moser LL, Whitley R, McHugo GJ, Bond GR, Finnerty MT, et al. Assertive community treatment: facilitators and barriers to implementation in routine mental health settings. Psychiatr Serv. 2009;60(2):189-95 https:// doi.org/10.1176/ps.2009.60.2.189.

91. Moser LL, DeLuca NL, Bond GR, Rollins AL. Implementing evidence-based psychosocial practices: lessons learned from statewide implementation of two practices. CNS Spectr. 2004;9(12):926-36 https://doi.org/10.1017/S1092 852900009780.

92. Powell BJ, Mandell DS, Hadley TR, Rubin RM, Evans AC, Hurford MO, et al. Are general and strategic measures of organizational context and leadership associated with knowledge and attitudes toward evidence-based practices in public behavioral health settings ? A cross-sectional observational study. Implement Sci. 2017;12(64):1-13.

93. Rapp CA, Etzel-Wise D, Marty D, Coffman M, Carlson L, Asher D, et al. Barriers to evidence-based practice implementation: Results of a qualitative study. Community Ment Health J. 2010;46(2):112-8 https://doi.org/10.1007/ s10597-009-9238-z.

94. Savill M, Skymba HV, Ragland JD, Niendam T, Loewy RL, Lesh TA, et al. Acceptability of psychosis screening and factors affecting its implementation: Interviews with community health care providers. Psychiatr Serv. 2018;69(6):689-95 https://doi.org/10.1176/appi.ps.201700392.

95. Van Erp NHJ, Giesen FBM, Van Weeghel J, Kroon H, Michon HWC, Becker D, et al. A multisite study of implementing supported employment in the Netherlands. Psychiatr Serv. 2007:58(11):1421-6 https://doi.org/10.1176/ps.2 007.58.11.1421.

96. Van Erp NHJ, Van Vugt M, Verhoeven D, Kroon H. Enhancing systematic implementation of skills training modules for persons with schizophrenia: Three steps forward and two steps back? Psychiatr Rehabil J. 2009;33(1):502 https://doi.org/10.2975/33.1.2009.50.52.

97. Williams NJ, Wolk CB, Becker-haimes EM, Beidas RS. Testing a theory of strategic implementation leadership, implementation climate, and clinicians ' use of evidence- based practice : a 5-year panel analysis. Implement Sci. 2020;15(10):1-15.

98. Aarons GA, Ehrhart MG, Farahnak LR, Sklar M, Horowitz J. Discrepancies in leader and follower ratings of transformational leadership: relationship with organizational culture in mental health. Adm Policy Ment Health Ment Health Serv Res. 2017;44(4):480-91 https://doi.org/10.1007/s10488-015-06727.

99. Aarons $G A$, Green $A E$, Willging $C E$, Ehrhart MG, Roesch $S C$, Hecht DB, et al. Mixed-method study of a conceptual model of evidence-based intervention sustainment across multiple public-sector service settings. Implement Sci. 2014;9(1):183 https://doi.org/10.1186/s13012-014-0183-z.

100. Bass BM, Avolio BJ. Multifactor Leadership Questionnaire (Technical Report). Binghampton; 1995.

101. Rogers EM. Diffusion of preventive innovations. Addict Behav. 2002;27(1): 989-93 https://doi.org/10.1016/50306-4603(02)00300-3.

102. Nelson TD, Steele RG. Predictors of practitioner self-reported use of evidence-based practices: Practitioner training, clinical setting, and attitudes toward research. Adm Policy Ment Health Ment Health Serv Res. 2007;34(4): 319-30 https://doi.org/10.1007/s10488-006-0111-x.

103. Gray MJ, Elhai JD, Schmidt LO. Trauma professionals' attitudes toward and utilization of evidence-based practices. Behav Modif. 2007;31(6):732-48 https://doi.org/10.1177/0145445507302877.

104. Beidas RS, Edmunds J, Ditty M, Watkins J, Walsh L, Marcus S, et al. Are inner context factors related to implementation outcomes in cognitive-behavioral therapy for youth anxiety? Adm Policy Ment Health Ment Health Serv Res. 2014;41(6):788-99 https://doi.org/10.1007/s10488-013-0529-x.

105. Nakamura BJ, Higa-Mcmillan CK, Okamura KH, Shimabukuro S. Knowledge of and attitudes towards evidence-based practices in community child mental health practitioners. Adm Policy Ment Health Ment Health Serv Res. 2011;38(4):287-300 https://doi.org/10.1007/s10488-011-0351-2.

106. Henggeler SW, Chapman JE, Rowland MD, Halliday-Boykins CA, Randall J, Shackelford J, et al. If you build it, they will come: Statewide practitioner interest in contingency management for youths. J Subst Abus Treat. 2007; 32(2):121-31 https://doi.org/10.1016/j.jsat.2006.07.006.

107. Hong Y, Liao H, Hu J, Jiang K. Missing link in the service profit chain: a meta-analytic review of the antecedents, consequences, and moderators of service climate. J Appl Psychol. 2013;98(2):237-67 https://doi.org/10.1037/a 0031666.

108. Zohar D. The effects of leaderhsip dimensions, safety climate, and assigned priorities on minor injuries in work groups. J Organ Behav. 2002;23(1):75-92 https://doi.org/10.1002/job.130.

109. Dinh JE, Lord RG, Gardner WL, Meuser JD, Liden RC, Hu J. Leadership theory and research in the new millennium: Current theoretical trends and changing perspectives. Leadersh Q. 2014;25(1):36-62 https://doi.org/10.101 6/j.leaqua.2013.11.005.

110. Wang G, Oh I, Courtright SH, Colbert AE. Transformational leadership and performance across criteria and levels: A meta-analytic review of 25 Years of research. Gr Organ Manag. 2011;36(2):223-70 https://doi.org/10.1177/1 059601111401017.

111. Podsakoff M, Mackenzie SB, Moorman H. Transformational leader behaviors and their effects on followers' trust in leader, satisfaction, and organizational citizenship behaviors. Leadersh Q. 1990;1(2):107-42 https://doi.org/10.1016/1 048-9843(90)90009-7.

112. Martinez RG, Lewis CC, Weiner BJ. Instrumentation issues in implementation science; 2014. p. 1-9.

113. Podsakoff PM, MacKenzie SB, Lee JY, Podsakoff NP. Common method biases in behavioral research: a critical review of the literature and recommended remedies. J Appl Psychol. 2003;88(5):879-903 https://doi.org/10.1037/00219010.88.5.879.

114. Mackinnon DP, Fairchild AJ, Fritz MS. Mediation analysis. Annu Rev Psychol. 2007;58(Hebb 1966):593-614.

115. Mackinnon DP, Fairchild AJ. Current directions in mediation analysis. Curr Dir Psychol Sci. 2009;18(1):16-20 https://doi.org/10.1111/j.1467-8721.2009.01 598.x.

116. Gifford W, Graham ID, Ehrhart MG, Davies BL, Aarons GA. Ottawa model of implementation leadership and implementation leadership scale: mapping concepts for developing and evaluating theory-based leadership interventions. J Healthc Leadersh. 2017;9:15-23 https://doi.org/10.2147/JHL. S125558.

117. Dorsey S, Pullmann MD, Kerns SEU, Jungbluth N, Meza R, Thompson K, et al. The juggling act of supervision in community mental health: implications for supporting evidence-based treatment. Adm Policy Ment Health Ment Health Serv Res. 2017;0(0):1-15.

118. Bower P, Gilbody S. Stepped care in psychological therapies: access, effectiveness and efficiency. Narrative literature review. Br J Psychiatry. 2005; 186(JAN):11-7.

119. Stein LI. Alternative to mental hospital treatment. Arch Gen Psychiatry. 1980; 37(4):392-7 https://doi.org/10.1001/archpsyc.1980.01780170034003.

120. May CR, Johnson M, Finch T. Implementation, context and complexity. Implement Sci. 2016;11(1):1-12, 141 https://doi.org/10.1186/s13012-0160506-3.

121. Walumbwa FO, Lawler JJ, Avolio BJ, Wang P, Shi K. Transformational leadership and work-related attitudes: The moderating effects of collective and self-efficacy across cultures. J Leadersh Org Stud. 2005;11(3):2-16 https://doi.org/10.1177/107179190501100301.

122. Zhu W, Avolio BJ, Walumbwa FO. Moderating role of follower characteristics With transformational leadership and follower work engagement. Gr Organ Manag. 2009;34(5):590-619 https://doi.org/10.1177/1059601108331242.

123. Lajoie D, Boudrias J-S, Rousseau V, Brunelle É. Value congruence and tenure as moderators of transformational leadership effects. Leadersh Org Dev J. 2017;38(2):254-69 https://doi.org/10.1108/LODJ-04-2015-0091.

124. Egeland KM, Skar A-MS, Endsjø M, Laukvik EH, Bækkelund H, Babaii A, et al. Testing the leadership and organizational change for implementation (LOCI) intervention in Norwegian mental health clinics: a stepped-wedge cluster randomized design study protocol. Implement Sci. 2019;14(1):1-12.

\section{Publisher's Note}

Springer Nature remains neutral with regard to jurisdictional claims in published maps and institutional affiliations. 